\title{
Magnitude judgments of loudness change for discrete, dynamic, and hybrid stimuli
}

\author{
Richard E. Pastore $\cdot$ Jesse Flint
}

Published online: 23 November 2010

(C) Psychonomic Society, Inc. 2010

\begin{abstract}
Recent investigations of loudness change within stimuli have identified differences as a function of direction of change and power range (e.g., Canévet, Acustica, 62, 2136-2142, 1986; Neuhoff, Nature, 395, 123-124, 1998), with claims of differences between dynamic and static stimuli. Experiment 1 provides the needed direct empirical evaluation of loudness change across static, dynamic, and hybrid stimuli. Consistent with recent findings for dynamic stimuli, quantitative and qualitative differences in pattern of loudness change were found as a function of power change direction. With identical patterns of loudness change, only quantitative differences were found across stimulus type. In Experiment 2, Points of Subjective loudness Equality (PSE) provided additional information about loudness judgments for the static and dynamic stimuli. Because the quantitative differences across stimulus type exceed the magnitude that could be expected based upon temporal integration by the auditory system, other factors need to be, and are, considered.
\end{abstract}

Keywords Loudness · Dynamic auditory stimuli .

Looming · Magnitude judgment

\section{Introduction}

In contrast to traditional studies that evaluated relative loudness for discrete changes in power across pairs of static (stationary)

\section{J. Flint}

State University of New York at Binghamton (SUNY),

NY Binghamton, USA

\section{R. E. Pastore $(\bowtie)$}

Department of Psychology, Binghamton University,

Binghamton, NY 13902-6000, USA

e-mail: pastore@binghamton.edu stimuli, recent investigations have evaluated change in loudness associated with stimuli that systematically vary in power. Canévet (Canévet \& Scharf, 1990; Canévet, Scharf, \& Botte, 1983; Canévet, Scharf, Teghtsoonian, \& Teghtsoonian, 1999; Teghtsoonian, Teghtsoonian, \& Canévet, 2000; Schlauch 1992) investigated changes in the perception of loudness associated with exposure to relatively long duration stimuli that systematically increased or decreased in power. With different goals, Neuhoff (1998, 2001) investigated loudness change across single, shorter duration stimuli that also varied systematically in power. In these studies, perceived change in loudness depended upon both direction of power change and either the average or ending magnitude (e.g., Canévet \& Scharf, 1990; Neuhoff, 1998, 2001; Teghtsoonian, Teghtsoonian, \& Canévet, 2005). Attributing his own results to the importance of looming, Neuhoff (1998) asserted that "None of these findings is predicted by traditional psychophysical laws derived using static stimuli, indicating that there are dramatic differences between static and dynamic loudness perception" (p. 123), but provided no basis for this claim. Furthermore, a careful, definitive empirical evaluation of the looming conjecture would be difficult to achieve, and neither of the experiments in the current study was intended to test such a hypothesis. Rather, the current study was designed to address several problems with the strong claim about contrast with past studies, specifically whether there are major differences in loudness perception for dynamic versus static stimuli.

First, Neuhoff (1998) did not justify his claim by citing either the relevant traditional studies or any specific psychophysical laws. Because Stevens' Power Law is widely cited, one might interpret the Neuhoff assertion as claiming that static, but not dynamic, power changes obey this relationship. However, this law is not universally accepted, and testing the law for dynamic or any other types of stimuli would have required Neuhoff to use a ratio 
(rather than interval) scaling procedure (see below). Teghtsoonian et al. (2005) used ratio scaling procedures to evaluate the validity of the Neuhoff findings with dynamic stimuli of equivalent duration, and found both similarities and differences (see below). Second, Neuhoff (1998, 2001) did not provide any empirical comparison of loudness change across dynamic versus discrete power changes, let alone an evaluation conducted with equivalent power changes using identical procedures, and no such empirical comparisons exist. Since there are continuing debates about the nature and validity of "traditional psychophysical laws derived using static stimuli" (Neuhoff, 1998, p. 123) about the loudness-power relationship, and Neuhoff (1998) provided no information about his assumptions about this relationship, his claim is, at best, fuzzy. The important current consideration is not finding a resolution to the long, complicated historical debate about the appropriate psychophysical relationship. Rather, it is the need for the empirical comparisons across stimulus type that can determine whether there actually are differences in loudness perception across static and dynamic stimuli and, if so, to identify the actual nature of these differences. The current Experiment 1 directly addresses the first aspect of this crucial, complex question, examining loudness change across four different types of stimuli, and finds consistent results across the various stimulus types. Then, as an attempt to better understanding the Experiment 1 results, Experiment 2 evaluates a form of loudness Points of Subjective Equality (PSE) for the prototypical static and dynamic stimuli investigated in Experiment 1.

\section{Loudness for discrete stationary stimuli}

Loudness is a complex subjective attribute of sound that is not equivalent to power, relative power, or $\mathrm{dB}$ change (e.g., Stevens \& Davis, 1938). Stevens' power law was developed over decades of investigation as a general psychophysical relationship that applied to most (i.e., prothethic or summative), but not all (i.e., metathetic or substitutive), sensoryphysical relationships (e.g., Stevens, 1935, 1955, 1956, 1961). It describes a ratio relationship that, for stimuli with a fixed power difference and spectral properties, is characterized by the exponent of the power function. The ratio relationship characterized by a specific power function should be independent of both average power (magnitude) and direction of power change. Although it is fairly typical to find descriptions of the application of this law to the mapping between loudness and power (e.g., Hartmann, 1998; Moore, 2004), this relationship has faced challenges. For example, Poulton (1984) argued that loudness for octave band noise stimuli reflects the combination of Fechner's law (thus an interval scale) altered by strong response bias, but this assertion was strongly rebuked by Teghtsoonian and
Teghtsoonian (1986). The relationship between loudness and power also has been variously argued to possibly reflect a discriminability scale (Garner, 1958) or even the judgment of distance (Warren, 1958, 1981). The Stevens' power law relationship has also been argued on occasion to reflect more about the individual's use or understanding of numbers than a reflection of an actual physical-perceptual relationship (e.g., Warren, 1981; Zwislocki \& Goodman, 1980). However, if loudness does approximate a prothetic continuum (e.g., Stevens, 1961), with the loudness-power relationship at least roughly corresponding to a power function, then, independent of stimulus properties, the relationship between loudness and power across stimulus type needs to be investigated using a ratio scaling procedure, rather than the interval scaling employed by Neuhoff (1998).

Even if valid, Stevens' power law would capture only part of the complex relationship between loudness and the physical properties of sound. The typically reported power law exponent for loudness is 0.6 for sound pressure and 0.3 for sound power, but this exponent value is specific to 1,000 Hz tone stimuli (Stevens, 1955; Zwicker \& Fastl, 1990). For constant power, loudness is a function of frequency (e.g., Fletcher \& Munson, 1933), with the power law exponent varying relatively systematically across frequency (e.g., Stevens, 1935). Furthermore, for broadband stimuli, loudness summates across critical bands (e.g., Zwicker, Flottorp, \& Stevens, 1957), resulting in a broadband noise power function exponent of only 0.23 (Zwicker \& Fastl, 1990). In addition, the loudness-power relationship, like any subjective judgment, is not invariant. Humans are known to develop and use subjective anchors (e.g., Braida \& Durlach, 1972; Durlach \& Braida, 1969; Helson, 1947), and this type of perceptual strategy might result in perceived magnitude being a function of some power average or range, and thus possibly direction of power change, especially when evaluated in separate blocks of trials (e.g., Poulton, 1968, 1969).

Both loudness and effective power also vary as a function of duration. Measurements of physical power reflect the RMS amplitude across a period of time. For example, power measured on a sound level meter is the RMS amplitude across a standard period that is either $1 \mathrm{sec}$ (Slow setting), $125 \mathrm{~ms}$ (Fast setting), or $35 \mathrm{~ms}$ (Impulse setting). Likewise, the auditory system integrates energy across time, with detection thresholds decreasing and perceived loudness increasing with increasing stimulus duration, at least up to durations of approximately 100300 ms (e.g., Hartmann, 1998; Moore, 2004; Yost, 2007). Although temporal integration (a concept further developed below) has important implications for the present study, as well as any study of loudness for stimuli that differ in temporal pattern of power across time, it would not be an important concern for the past investigations (described in 
the next section) that evaluated change in loudness for only a fixed $\mathrm{dB}$ change across a single type of dynamic stimulus. The important point here is that the many known relationships between loudness and the physical properties of stationary stimuli undermine the notion that there are simple, straightforward predictions from "traditional psychophysical laws derived using static stimuli." This, in turn, reinforces the need for the direct empirical comparisons across static and dynamic stimuli under equivalent conditions, and thus the current study.

\section{Loudness for stimuli dynamically changing in power}

Canévet and colleagues (e.g., Canévet, 1986; Canévet \& Scharf, 1990; Canévet et al., 1983; Canévet et al., 1999) used standard ratio scaling to investigate changes in loudness associated with exposure to relatively systematic, long duration changes in power. For example, Canévet (1986) found that systematic decreases in power resulted in decruitment or sweep-induced fading of loudness near the end of long ( $3 \mathrm{~min}$ ) stimuli, but analogous changes were not found for equivalent increases in power. Such changes were attributed to a form of adaptation.

Neuhoff (1998) evaluated the perceived change in loudness of linear $15 \mathrm{~dB}$ increases and decreases in power across the $1,800 \mathrm{~ms}$ duration of three different types of acoustic signals $(1,000 \mathrm{~Hz}$ tone, white noise, and a synthetic vowel), with onset and offset power ranging between 60 and $90 \mathrm{~dB}^{1}$. Participants indicated loudness change on an undifferentiated interval displayed on a computer monitor, and thus an interval (or possibly only ordinal) scale. The data indicated that, for equivalent average power, perceived loudness change is nearly always greater for increasing than decreasing power, and this pattern of results was attributed to the biological importance of looming. Finally, independent of change directions, perceived loudness change is always greater for more intense stimuli. However, there are serious problems in trying to use interval scaling to evaluate presumed ratio relationships (e.g., Stevens, 1961). As an example, using the power exponent typically reported for the $1,000 \mathrm{~Hz}$ tone stimuli used by Neuhoff, the $15 \mathrm{~dB}$ increases and decreases in power respectively should represent 2.8:1 increases and 1:0.35 decreases in loudness that, although equal opposite ratios, are not equal subjective intervals. If the underlying relationship is ratio, then measuring subjective loudness changes using an interval scale should result in apparent greater changes for power increases than decreases. In addition, the ordering of loudness change across stimulus type reported by Neuhoff (1998) is actually consistent with

\footnotetext{
${ }_{1}^{1}$ Although not specified by Neuhoff (1998), we assume that intensity is in either $\mathrm{dB}$ :SPL or $\mathrm{dB}(\mathrm{C})$.
}

the power law exponents for a $1,000-\mathrm{Hz}$ tone, a broad band noise, and the dominant low frequency component of a harmonic series (see above) ${ }^{2}$. Despite criticisms of the methodology in this original Neuhoff study (e.g., Canévet et al.,1999; Hall \& Moore, 2003; Teghtsoonian et al., 2005), a subsequent study by Neuhoff (2001) used the same methodology and, not surprisingly, obtained similar results.

A major goal of the Teghtsoonian et al. (2005) study was an attempt to reconcile the differences across the Neuhoff (1998) and several Canévet studies (Canévet, 1986; Canévet \& Scharf, 1990; Canévet, Teghtsoonian, \& Teghtsoonian, 2003) for dynamic changes in power with and without a discrete initial stationary (constant-power) component or plateau. Teghtsoonian et al. used ratio scaling to investigate perceived loudness change for the $1,800 \mathrm{~ms}$, $1,000 \mathrm{~Hz}$ stimuli across a $30-90 \mathrm{~dB}: \mathrm{SPL}$ range that was double the $60-90 \mathrm{~dB}$ range investigated by Neuhoff. For the two contrasted magnitudes of power change (15 or $30 \mathrm{~dB}$ ), the magnitude of loudness change for both change directions was a function of the terminal power of the stimulus, but more so for increasing power. Thus, consistent with the interval-based Neuhoff results, these ratiobased estimates of change in loudness were dependent upon stimulus intensity. However, Teghtsoonian et al. (2005) found a directional dependency inconsistent with a strict interpretation of both the Neuhoff results and Stevens' power law. Specifically, a 2:1 change in power (30 vs $15 \mathrm{~dB}$ ) resulted in a 2:1 (thus, $100 \%$ ) change in loudness for decreasing power, but only an $18 \%$ change for increasing power. Finally, these authors favor a sensory or psychophysical, rather than an evolutionary or Darwinian, explanation.

\section{Current study}

The primary focus of Experiment 1 is a broad, multipart question about loudness as a function of stimulus power envelope. The first part of the question is whether there are differences in the perception of loudness change for discrete stationary, dynamic, and two theoretically intermediate or hybrid amplitude envelopes of a $1,000-\mathrm{Hz}$ stimulus when methodology is common to, rather than confounded with, stimulus type. The second part of the question is whether there are differences in the perception of change in loudness

\footnotetext{
${ }^{2}$ The predictions for the $1,000-\mathrm{Hz}$ and noise stimuli are straightforward. For typical synthetic vowel stimuli, energy is greatest at the lowest frequency; but the overall power of these stimuli would depend upon the (unspecified) dB scale used by Neuhoff to specify power, as the $\mathrm{dB}$ scales differ primarily in the low frequency region. In addition, the contribution of the different frequency components to overall loudness should have varied as a function of overall power, as the rate of growth in loudness differs across frequency (e.g., Fletcher \& Munson, 1933).
} 
that are dependent on the direction of change in power. The answers to this multipart question have important implications for the current debate about the scaling of static versus dynamic stimuli, as well as directions of future investigations about the mapping between loudness and power as a function of stimulus type. In principle, change in loudness for any of the stimulus types should reflect a loudness comparison of the estimated magnitudes of the initial and terminal portions of the given stimulus. Following this logic, the goal of Experiment 2 is to achieve an understanding of the qualitative and quantitative patterns of Experiment 1 based upon estimates of the perceived magnitude (PSE) of the onset and offset aspects of the prototypical Static and Dynamic stimuli evaluated in Experiment 1.

\section{Experiment 1}

There are three main goals within the broad question addressed in Experiment 1. One is to evaluate loudness change across the dynamic power changes in the $1,000-\mathrm{Hz}$ tone stimuli described in Neuhoff (1998), but using a ratio, rather than interval, scaling procedure. Although this goal was met by Teghtsoonian et al. (2005), the remaining goals of the current experiment can only be met by evaluating all stimulus types using the identical procedure. Because Neuhoff (1998) did not provide any literature-based comparison, let alone a direct comparison, with nominally equivalent power change across stationary stimuli, a second goal is to provide this empirical comparison. The third goal is to attempt to determine what aspect of the stimuli might be responsible for any differences across stimulus type or direction of power change. This goal was addressed by evaluating the two conceptually intermediate or hybrid stimuli that differ in the discrete versus dynamic nature of the central portion of the stimulus.

All auditory stimuli were $1,000-\mathrm{Hz}$ tones with a total duration of $1,800 \mathrm{~ms}$, and power within the $60-90 \mathrm{~dB}(\mathrm{C})$ range most likely studied by Neuhoff (1998), but with 10-, $15-$, and $20-\mathrm{dB}$ changes in power, rather than only the $15-\mathrm{dB}$ change investigated by Neuhoff (1998) or the 15- and 30-dB changes investigated by Teghtsoonian et al. (2005). The four different types of auditory stimuli investigated in the current study (Dynamic, Static, Hybrid-Static and Hybrid-Dynamic) are illustrated in Fig. 1, but only for increasing changes in power; the equivalent decreasing power stimuli can be illustrated by the temporal (abscissa) or power (ordinate) reversal of the stimuli in this figure.

The Dynamic stimulus (Fig. 1a) was investigated by Neuhoff (1998). The Static stimulus (Fig. 1b) is the stationary, nominally equivalent (in terms of power and power change) of the Dynamic stimulus, but represents the

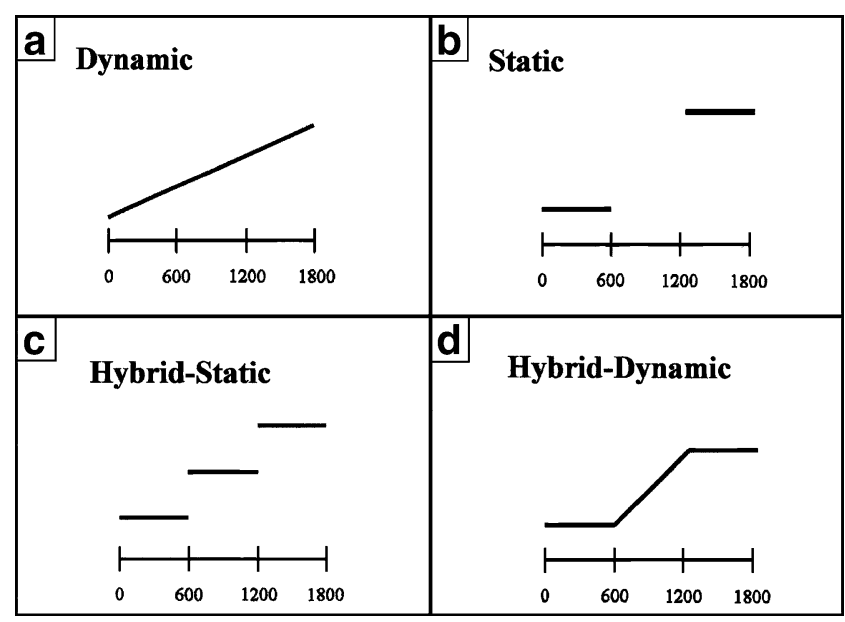

Fig. 1 Chart illustrating the types of $1,800-\mathrm{ms}, 1,000-\mathrm{Hz}$ auditory stimuli used in Experiment 1. The stimuli reflect the conditions with increases in power across the 1,800-ms stimulus duration. For each stimulus type, decreases in power would be illustrated by reversing the stimuli along either the horizontal (time) or vertical (power) axis

type of stimulus investigated by Stevens (e.g., 1956). If discrete stationary and dynamic stimulus changes are processed differently, there should be qualitative differences in the pattern of loudness judgments across these two stimulus types. However, whether or not the stimuli are processed in an equivalent manner, quantitative differences should be found. Specifically, the effective power of a stimulus is not the physical rms amplitude over a few ms, but rather the result of temporal integration by the auditory system. Thus, although the initial and final few ms of these two stimulus types may be equivalent in physical amplitude, temporal integration by the auditory system (as well as the setting of any sound level meter) creates a difference in effective initial and final power across these two stimulus types. Whether the auditory system's integration window is as short as 100 or as long as 300 ms (e.g., Moore, 2004; Yost, 2007), the effective power of the initial and final (600-ms) segments of the Static stimuli, and thus the change in effective power across these segments, are appropriately characterized by the physical specifications. Thus, at least in principle, the Static stimuli should result in loudness ratios that reflect Stevens' power law with the 0.3 exponent typical of $1,000-\mathrm{Hz}$ tones, independent of direction of change.

For any Dynamic stimulus, power is systematically changing across the auditory system's temporal integration windows located at stimulus onset and offset. Relative to the nominal physical power at onset and offset, the effective or integrated power of the initial and final portions of a Dynamic stimulus depends upon the rate of power change and the duration of the integration window, but will always be regressed somewhat toward the average or median power of the stimulus. For example, relative to a Static 
stimulus with nominally identical onset and offset power, a Dynamic stimulus with increasing power (illustrated in Fig. 1a) will have greater effective power at onset, and lower effective power at offset, and thus a smaller change in effective power. If loudness change is determined, even if only in part, by change in effective power, then there should be a quantitative difference in loudness change across these two stimulus types, with the magnitude of difference dependent on the characteristics of the effective auditory system integration window.

The two Hybrid stimuli are illustrated in the bottom panels of Fig. 1. The Static and two Hybrid stimuli all have three equal (600-ms) duration segments that, with identical initial and final static segments, are distinguished solely by the nature of the middle segment. The middle segment in the Static stimuli (Fig. 1b) is a silent interval, but is a 1,000$\mathrm{Hz}$ tone in the two Hybrid stimuli. In the Hybrid-Static stimuli (Fig. 1c), the middle segment is static with power (in $\mathrm{dB}$ ) that is midway between the initial and final segments. With two equal $\mathrm{dB}$ changes, if an approximation of Stevens' power law applies, there should be two roughly equal changes in loudness across the three segments of these Hybrid-Static stimuli. For the Hybrid-Dynamic stimuli (Fig. 1d), the middle segment is a dynamic linear change in $\mathrm{dB}$ from the initial to final segments. For the stimuli in Fig. 1b, c and d, because the initial and final segments are identical and considerably longer than typical estimates of the human integration window, any qualitative or quantitative differences in loudness change across these three stimulus types must be due to the nature of the middle component. Although the four stimulus types investigated do not control for all possible factors (e.g., the velocity of power change within stimuli does differ across stimulus type), their investigation does provide the basis for an initial evaluation of the effects on loudness judgments of (1) static versus the specific dynamic power change (Static versus Dynamic stimuli), (2) whether or not there is an intervening stimulus power magnitude (Static versus the other three stimulus types), and (3) whether that intervening component is discrete or dynamic (Hybrid-Static versus Hybrid-Dynamic), as well as (4) whether power change directional differences depend upon stimulus type.

The task of the listener was to judge the relative magnitude of change in loudness between the onset (or initial) and offset (or final) portions of the stimuli. Using the ratio scaling literature (e.g., Stevens, 1956; Stevens \& Poulton, 1956; Warren, 1981) and pilot conditions for the current study as guides, the perceived initial magnitude of each stimulus was treated as the reference or standard, with the listener judging the magnitude of the final portion of the stimulus relative to that reference. The task thus was to judge the loudness ratio between perceived offset and onset power, and thus the change in loudness across the stimulus.
Because of classic concern that bias in the use of numbers might truncate estimated magnitude (e.g., floor or ceiling effects in use of numbers), the initial magnitude reference was always assigned the commonly used numerical value of 100 (Poulton, 1969; Teghtsoonian et al., 2000, 2005; Warren, 1981).

To expose participants to a ratio magnitude judgment tasks, as well as to screen for participants who lack an understanding of, or motivation to understand, ratio magnitude judgments, a visual scaling condition was run prior to the auditory loudness conditions. Utilizing the same scaling procedure, participants judged the area of a comparison circle relative to that of a circle reference whose area varied across trials, with the reference always assigned a magnitude value of 100 . Teghtsoonian (1965) had studied the scaling of circle area and size, and Teghtsoonian et al. (2000) subsequently used circles of dynamically changing area in a magnitude estimation task to demonstrate decruitment in a different modality.

\section{Methods}

\section{Participants}

All participants in this (two-experiment) study were from the Binghamton University Psychology Department subject pool and volunteered as fulfillment of an alternative partial requirement for an undergraduate course. There were a total of 112 participants in Experiment 1. Each participant performed the identical circle scaling task, then the loudness scaling task for one of four auditory conditions defined in terms of stimulus type (i.e., Dynamic, Static, Hybrid-Static, or Hybrid-Dynamic, as illustrated in Fig. 1). The circle scaling task thus did not differ across auditory conditions and provided the a priori criterion for participant inclusion in this experiment. If a participant's circle judgment data indicated a lack of understanding of the ratio judgment scaling task (e.g., responded with numbers below 100 for areas greater than the reference, or with the minimal difference in estimated magnitude despite large differences in area ratio), that participant was dropped from the study and an additional participant was run. Based upon this a priori circle scaling criterion, 24 participants were dropped across the four conditions, leaving 22 participants in each group.

\section{Stimuli}

Visual circle A series of five circles differing in area (3.55, 5.3, 7.1, 10.6, and $14.2 \mathrm{~cm}^{2}$ ) were created in Publisher (Microsoft). Circle area was measured as viewed on a 15inch $(\mathrm{c} .38 .1-\mathrm{cm})$ computer monitor; participant viewing distance was approximately $45 \mathrm{~cm}$. On each trial, a pair of 
circles was presented simultaneously, with the circle on the left always being the reference standard and always explicitly assigned a magnitude of 100 . The comparison circle was always presented on the right and always differed from the standard. The pair of circles was defined in terms of both the "circle area ratio" (e.g., 7.1/3.55=2.0), which did not depend upon viewing distance, and "mean circle area" (e.g., 7.1 vs $3.55=5.325$ ). The labels "larger" and "smaller" are used to reflect the size of the comparison (right) circle relative to the reference (left) circle.

Auditory All stimuli were $1,000-\mathrm{Hz}$ sinusoids synthesized at a 16-bit, $44.1-\mathrm{kHz}$ sampling rate $(10 \mathrm{~ms}$ onset and offset ramps) using Audition 2.0 (Adobe) and presented binaurally through Sennheiser Model HD 265 headphones. Table 1 lists the nominal onset and offset power $\mathrm{dB}(\mathrm{C})$ and power change $(10,15$ and $20 \mathrm{~dB})$ for all stimulus comparisons investigated. Every auditory stimulus was defined in terms of power at onset and offset as well as median power (e.g., the 65 - to $85-\mathrm{dB}(\mathrm{C})$ stimulus has a 20 $\mathrm{dB}$ change in power and a median intensity of $75 \mathrm{~dB}$ ). Although Teghtsoonian et al. (2005) analyzed their data in terms of final intensity, the various properties of the $\mathrm{dB}$ specifications of the current stimuli (initial, median and final intensity) are perfectly correlated within and partially correlated across the three magnitudes of $\mathrm{dB}$ change. Because physical power measures reflect rms power across a period of time, rather than the instantaneous value specified in a synthesis program, the onset, offset, and $\mathrm{dB}$ change values specified in Table 1 for the Dynamic stimuli are only nominal approximations and are not perfectly equivalent to the other three stimulus types. Following the same logic, auditory system temporal integration means that the effective magnitude of the initial and final aspects of Dynamic stimuli (Fig. 1a), although nominally equivalent to the other stimulus types (Fig. 1b, c and d), will differ in effective power, and thus have smaller effective power change. Without assumptions about the appropriate duration of the temporal integration window, the most accurate designation of both physical and effective power across the current four stimulus types is the median power statistic. This descriptive property provides information that is directly related to the nominal final stimulus intensity and also allows a specification that can be used to compare stimuli that differ in $(10-, 15-$ and $20-\mathrm{dB})$ change magnitudes.

\section{Procedure}

All participants in the current study were tested in a commercial soundproof booth. Each session began with the identical pair of circle area judgment tasks that differed
Table 1 Summary of power attributes of the stimuli investigated in Experiment 1

\begin{tabular}{llll}
\hline dB Range & Increasing & Decreasing & Median \\
\hline $20 \mathrm{~dB}$ & $70-90$ & $90-70$ & 80 \\
& $65-85$ & $85-65$ & 75 \\
& $60-80$ & $80-60$ & 70 \\
$15 \mathrm{~dB}$ & $75-90$ & $90-75$ & 82.5 \\
& $70-85$ & $85-70$ & 77.5 \\
& $65-80$ & $80-65$ & 72.5 \\
& $60-75$ & $75-60$ & 67.5 \\
$10 \mathrm{~dB}$ & $75-85$ & $85-75$ & 80 \\
& $70-80$ & $80-70$ & 75 \\
& $65-75$ & $75-65$ & 70 \\
\hline
\end{tabular}

Onset, offset, and median power (dB:C) and power change (10, 15 and $20 \mathrm{~dB}$ ) for all stimulus comparisons

only in the standard-comparison area relationship (larger versus smaller, thus numerical judgments greater versus less than 100), with task order counterbalanced across participants. For the subsequent auditory task, the participant judged only one of the four stimulus types (Dynamic, Static, Hybrid-Static, or Hybrid-Dynamic), again with separate, blocked evaluations of increasing and decreasing power, ${ }^{3}$ with block order (e.g., numerical judgments greater versus less than 100) matched to that in the circle task. Thus, the four blocks of scaling tasks run by half the participants was the larger, then smaller circle area judgment followed by the increasing, then decreasing power loudness judgment, with the other half of participants running the small then large circle followed by the decreasing, then increasing power loudness judgment tasks. In each of the four blocks of trials, there were four presentations of each stimulus pair, for a total of 80 trials in each modality.

The nature of the scaling tasks was explained to participants both during and following informed consent. Once the participant entered the booth, all aspects of the experiment (instructions, stimulus ordering and presentation, etc.) were controlled by an E-Prime program (Psychology Software Tools), with all instructions, as well as the circle stimuli, displayed on the monitor. On each trial, instructions indicated that one stimulus (left for circles, initial or onset portion for auditory) was the standard and had a magnitude value of 100 . The participant was

\footnotetext{
${ }^{3}$ Teghtsoonian et al. (2000) found that multiple judgments along a continuously decreasing or continuously increasing scale resulted in power functions with increased slopes or accelerated shrinkage. Because the stimuli in Experiments 1 and 2 were randomized within increasing or decreasing power, participant judgments are not continually increasing or decreasing across trials, and thus, should not be affected by accelerated shrinkage.
} 
instructed to assign a number indicating the magnitude of the other stimulus (right for circles, final or offset portion for auditory) relative to the standard. Participants were given as much time as needed to respond, typing the digits on the number pad of a standard computer keyboard (with corrections permitted), then hitting the "Enter" key to record their response. No feedback was provided.

Results and discussion

Measures of central tendency across the four presentations of each stimulus pair were computed for each participant. With similar patterns and magnitudes of results for median and geometric mean values in both the circle and sound conditions, we report only the geometric means. In addition, because initial analysis of all of the data indicated equivalence across the order of judgment trial blocks run (larger/ smaller circle area, increasing/decreasing sound power), the data were pooled across this variable, with order not considered in the reported data analyses. Judgments were transformed relative to unity, with judgments relative to a larger standard (i.e., ratios <1) also inverted to allow comparison of directional differences on the same scale. Because the data were ratio, not interval, all ANOVAs were conducted on the logarithm of the results.

\section{Circle area}

The circle stimulus results were considered to be a direct indication of each participant's use of the ratio scaling methods to reflect estimations of relative magnitude. Because condition was defined solely by the nature of the auditory stimulus type, the circle results should not, and do not, differ across condition, $F(3,84)=1.83, p=0.15, \dot{\eta}^{2}=$ .06 , indicating a lack of difference in judgments across participant groups.

A power function plotted in log coordinates is a straight line with slope equal to the exponent. Geometric mean area ratio estimates across participants for each stimulus comparison are plotted in the logarithmic coordinates of Fig. 2 as a function of physical area ratio. Because pairs of stimuli with identical physical area ratios, but different average areas, tended to result in very similar ratio judgments, abscissa values were altered slightly (ordinally as function of area) to improve the visibility of individual data points (thus slightly reducing apparent goodness of fit in Fig. 2). The solid line in this figure indicates equivalence between the actual and judged area ratios (i.e., a power function exponent of 1.0 with no systematic bias). Both the larger (filled squares) and smaller (open circles) judgment area relationships are linear $\left(r^{2}=0.99\right)$ in the log coordinates of Fig. 2, indicating power law relationships. Furthermore, as is typically reported in the literature (e.g., Teghtsoonian,
1965), the exponents are less than 1 . Thus, those participants included in the loudness portion of Experiment 1 were able to use the ratio scaling methods and essentially replicated earlier results for the circle stimuli.

\section{Sounds}

The geometric mean loudness ratio results are summarized in Fig. 3 as a function of median dB power (abscissa), dB change (respectively, 20,15, 10 as separate columns of panels), direction of change (2 rows of panels) and stimulus type (symbols). The first question is whether there are fundamental differences in loudness perception across the dynamic and stationary (static) stimulus types. To better visually compare the results for the four stimulus types across the different power changes, the scale of the ordinate for the $10 \mathrm{~dB}$ change (right set of panels) has been expanded. The simple answer to the dynamic-static question, immediately apparent in Fig. 3, is negative; the qualitative pattern of results does not differ across the Static versus Dynamic (solid lines with filled symbols), or even across any of the stimulus types investigated. The second major question is whether there are differences in loudness change perception as a function of direction of power change. Again, it is clearly evident in Fig. 3 that there are major qualitative differences as a function of direction of power change (upper versus lower panels), and the differences again are qualitatively consistent across stimulus type and $\mathrm{dB}$ change. The full answers to these questions, however, are complicated. First, inferential statistics are designed to assign a probability to making an error by rejecting the null hypothesis, rather than an error by accepting the null hypothesis of no difference across both stimulus type, and the data patterns clearly seem to reflect no qualitative differences across stimulus type. Second, because of temporal integration by the human auditory system, there should be quantitative differences

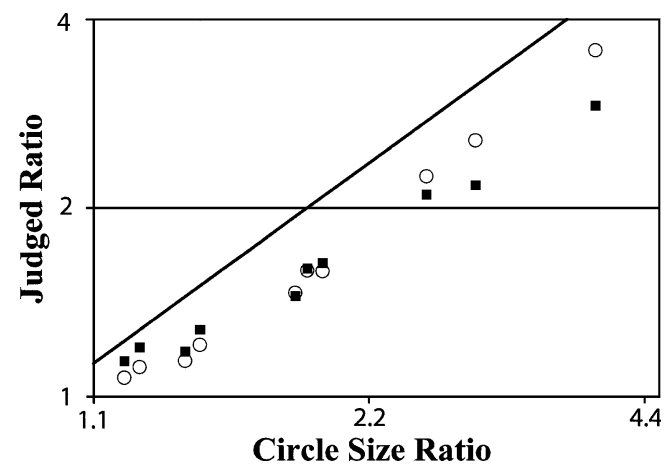

Fig. 2 Geometric mean of size ratios for each circle area comparison as a function of physical size ratio in logarithm coordinates. Open circles represent small-to-large comparisons. Filled squares represent large-to-small comparisons 

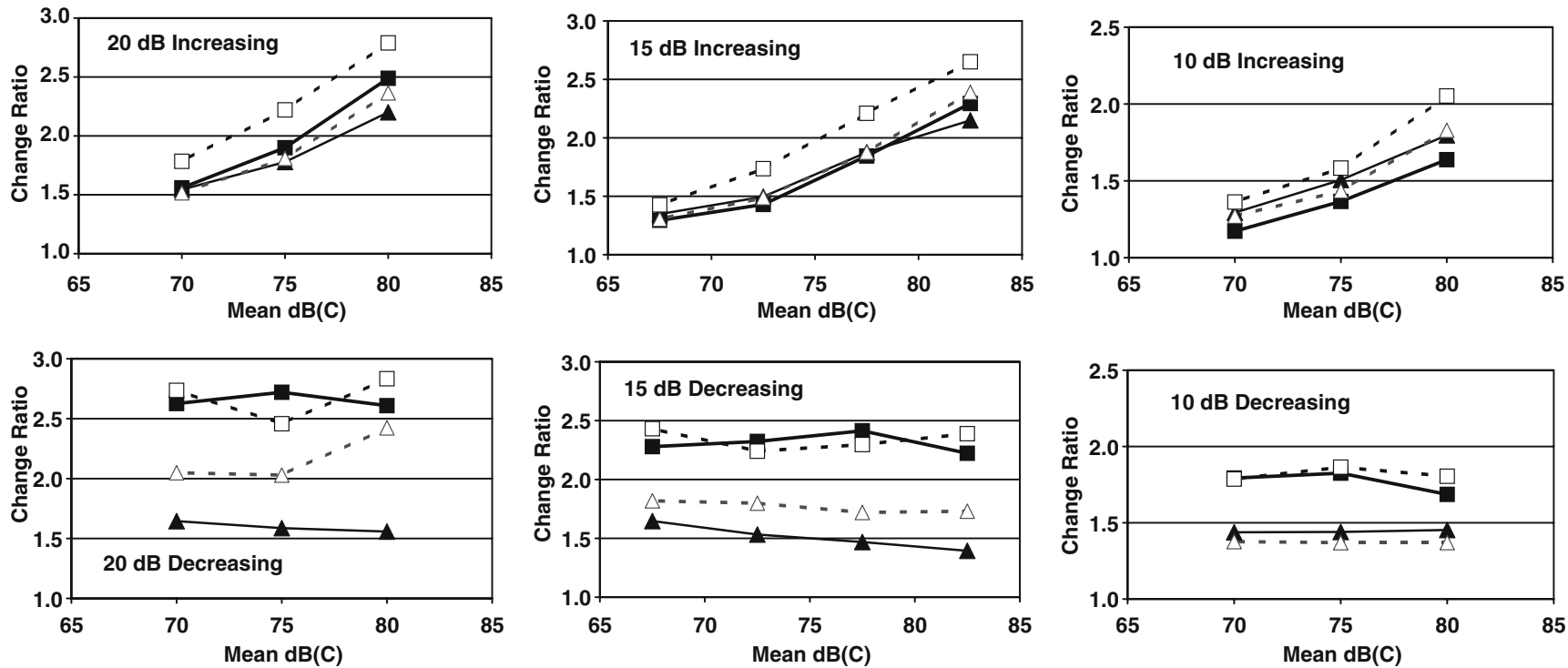

Fig. 3 Geometric mean loudness ratio results as a function of median $\mathrm{dB}(\mathrm{C})$ power. Left, middle, and right columns summarize results for $20 \mathrm{~dB}, 15 \mathrm{~dB}$, and $10 \mathrm{~dB}$ onset-offset differences in power (with ordinate scales differing as a function of $\mathrm{dB}$ change), respectively. The top row of panels summarizes the results for increasing power across time and the bottom row represents decreasing power across time. The

between the one Dynamic stimulus type and the three other stimulus types (with static onset and offset segments). Furthermore, the direction of these differences depends upon the direction of power change (i.e., for stimuli with nominally identical onsets, the effective power at the beginning and end of the $n$ ms integration window located precisely at onset will differ across direction of power change for the Dynamic stimuli, but should not differ across the other three types of stimuli evaluated). Thus, for the Dynamic stimuli, there is the expectation of differences in the magnitude of effective power change, and thus the magnitude of loudness change, whether or not there are any fundamental differences in the way loudness is perceived across the four stimulus types. These factors all complicate the more detailed analyses of the results.

At the suggestion of a reviewer, since the initial question for the current research concerns the claims about fundamental dynamic-static differences, the initial detailed analyses (solid lines in Fig. 3) focuses on the Static (square filled symbols, bold lines) and Dynamic (triangle filled symbols, light lines) stimulus types. For the increasing changes in power (upper panels), there is not a significant quantitative difference across these stimulus types, $F(1,42)=0.15, p=$ $.90, \dot{\eta}^{2}=0.05$. For the decreasing changes in power, there is a significant quantitative difference between the Static and Dynamic stimuli, $F(1,42)=42.67, p<.001, \dot{\eta}^{2}=1.00$. Furthermore, the magnitude of differences between these stimulus types is probably greater than would be expected based solely on the basis of a simple, ideal model of filled symbols with solid lines are for the prototypical static (Static; squares) and dynamic (Dynamic; triangles) stimuli. The open symbols with broken lines are for the hybrid stimuli with center portion static (Hybrid-Static; squares) and dynamic (Hybrid-Dynamic; triangles) stimuli

temporal integration (addressed below and in General discussion). Had only the current Static and Dynamic stimuli been investigated, the conclusion would have been that there is a major difference in processing generically defined dynamic and static stimuli when power is decreasing over time, thus supporting this aspect of the Neuhoff claim. However, the grouping of results in Fig. 3 is not between the judgment of stimuli with static versus dynamic initial and final components (i.e., Static, Hybrid-Static, and HybridDynamic versus Dynamic). Instead, the quantitative difference is largely between the stimuli with only static components (Static and Hybrid-Static; square symbols) and the stimuli with any dynamic component (Dynamic and Hybrid-Dynamic; triangle symbols), and the contrast between these two groupings is significant $F(1,86)=45.09, p<.01$, $\dot{\eta}^{2}=.34$. The implication is that, for decreasing power changes, the presence of a dynamic change in power within the stimulus alters judgment of loudness change.

The basis for the looming conjecture about loudness was the finding of greater changes in loudness for increasing than decreasing power changes. The second set of basic questions thus concern whether there are differences in the magnitude of loudness change as a function of overall stimulus magnitude and direction of power change. The answers to this set of questions are made complicated by the patterns of results. It is obvious in Fig. 3 that, for all four stimulus types, the magnitude of loudness change is a function of median (and thus overall) stimulus power when power increases over time (upper 
Fig. 4 Increasing-decreasing ratio of loudness change (increasing ratio/decreasing ratio) for each stimulus type (four panels), median $\mathrm{dB}$ value (abscissa) and $\mathrm{dB}$ change (symbols)
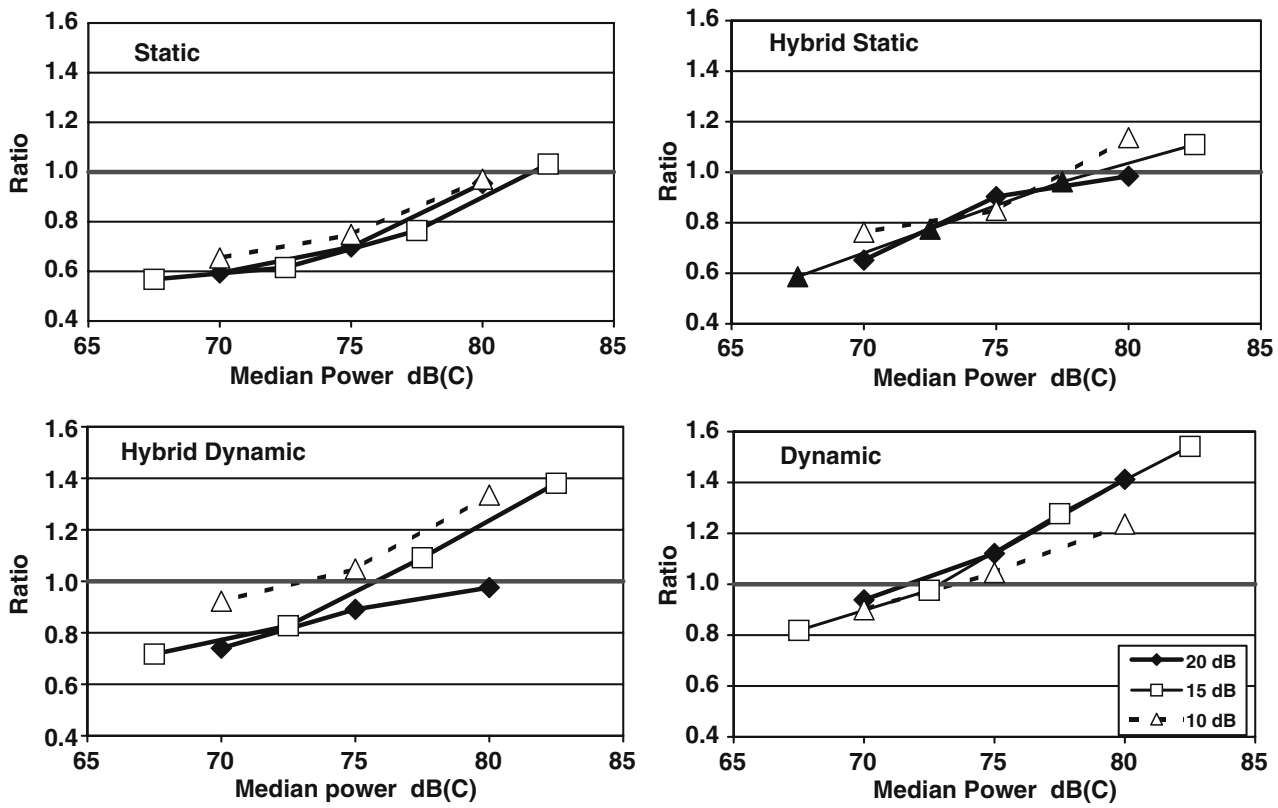

panels of Fig. 3), $F(2,85)=336.06, p<.001, \dot{\eta}^{2}=.80$, and this pattern of results is not consistent with Stevens' power law. In contrast, when power decreases over time (lower panels), loudness change is independent of median power, $F(2,85)=.302, p=.739, \dot{\eta}^{2}=.10$. This pattern of results is consistent with Stevens' power law. In addition, there are differences in magnitude of loudness change across $\mathrm{dB}$ change, average $(\mathrm{dB})$ magnitude, and stimulus type. These differences do not allow simple comparisons that reflect relative magnitude of loudness change across direction of power change, but such comparisons can be achieved by a simple transformation of the data.

A direct analysis of the importance of direction of power change is facilitated by computing an increasing-decreasing ratio of loudness change (increasing ratio / decreasing ratio) for each stimulus type, $\mathrm{dB}$ change, and median $\mathrm{dB}$ value (i.e., each data point in the upper panels of Fig. 3 divided by the corresponding point in the lower panels). Ratios greater than (versus less than) unity indicate that, for the given median power, equal $\mathrm{dB}$ changes result in greater loudness change for increasing (versus decreasing) stimulus power. These ratios are plotted in Fig. 4. For the stationary Static stimulus type, relative loudness change is a systematic function of median power and is greater for decreasing power (ratios less than 1.0) for all but the highest median stimulus power (where there is equality across direction of power change). Although somewhat more variable, the other three stimulus types exhibit a similar trend, but all with ratios both less than and greater than unity, and with the directional cross-over (ratio $=1.0$ ) progressing toward lower median power as a function of stimulus type. Thus, loudness change is not always greater for increasing than decreasing change in power. However, for the Dynamic stimuli, when power is above $75 \mathrm{~dB}$ :SPL, increasing power does result in greater change in perceived loudness decreasing power, and these results are consistent with those reported by both Neuhoff (1998) ${ }^{4}$ and Teghtsoonian et al. (2005). These results are a problem for strict generality of the simplest looming conjecture, as it would require something like a qualification that the conjecture applies only at higher power levels, levels that might indicate a potentially closer or more powerful source.

Finally, because the current study investigated loudness change across both discrete and dynamic changes power, it is important to consider the consequences of temporal integration by the auditory system in defining the effective power at the onset and offset of the different stimulus types, and thus the effective change in power across the full duration of each stimulus. What magnitude of difference in effective power might be expected due to solely temporal integration? For a fixed duration integration window, the difference in effective power between stationary and dynamic power changes would be a direct function of rate of power change. Assuming perfect power integration over the maximum estimated duration of the integration window (300 ms), the nominal 10-, 15-, and $20-\mathrm{dB}$ changes, although accurate for the Static and two Hybrid stimuli, would represent effective power changes of approximately $8.3,12.5$, and $16.7 \mathrm{~dB}$ for the Dynamic stimuli. Further assuming a power function exponent of 0.3 for the $1,000-\mathrm{Hz}$ stimuli, these changes in effective power for the Dynamic stimuli should result in loudness change ratios of 1.9, 2.1, and 2.3. Even if one assumed leaky (rather than

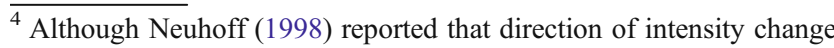
was not statistically significant, there is a trend in the data summarized in his Fig. 1 that is similar to that reported here.
} 
perfect) integration (e.g., Munson, 1947; Plomp \& Bouman, 1959), the effective power relationships, and thus the loudness ratios, probably would not change appreciably, meaning that integration efficiency alone is probably not important. Although the difference in effective power has to be a small factor in the judgments of loudness, the differences in loudness ratio between the Static and Dynamic decreasing power stimuli (Fig. 3, bottom panels) are much too large for temporal integration to be the only factor. Finally, for the increasing power changes, the Hybrid-Static stimuli always resulted in greater changes in loudness than the Static stimuli, and for the decreasing power changes, there is the already noted significant effect of the presence versus absence of a dynamic change in power within the stimulus. Thus, it is likely that, at a minimum, both temporal integration and the nature of an intervening stimulus component play roles in judgment of loudness change.

\section{Experiment 2}

In one very logical conceptualization, the measure of loudness change in Experiment 1 reflects, for each stimulus, a comparison of the effective (or subjectively estimated) power magnitude of the stimulus at offset relative to onset. Effective magnitude depends at a minimum upon some form of temporal integration, and thus a difference between Static and Dynamic stimuli with nominally equal physical onset and offset specifications. Since judgment of loudness change or ratio can only take place after the ending magnitude has been encoded, there must also be storage of some form of information about onset magnitude to allow the needed comparison. Whether the stored information directly represents integrated magnitude or a transformation into distance or some other variable, there is the possibility (and the Experiment 1 results suggest) that the stored information may be altered or disrupted by the subsequent portion of the stimulus. Whether or not the compared information about onset and offset magnitudes are accurate reflections of integrated physical magnitude, there is also the possibility of a processing or decision asymmetry between large-to-small and small-to-large judgments. An initial step toward differentiating among these and other possibilities is to measure the effective magnitude of power at stimulus onset and stimulus offset. In addition, the direct quantification of the perceived magnitudes of the onset and offset portions of stimuli might provide an indication of the contribution of temporal integration, temporal position within the stimulus, and other stimulus properties to the qualitative and quantitative results in Experiment 1. This is the logic underlying Experiment 2. It is implemented by measuring the Point of Subjective Equality (PSE) for loudness of the onset and offset portions of the Static and Dynamic stimuli. PSE is the physical magnitude of a reference stimulus that is subjectively equivalent to the targeted stimulus component. It is measured by judgment of the relative magnitude of the designated portion of the target stimulus and a reference stimuli presented on each trial.

In Experiment 1, there were quantitative differences among the Static and the two hybrid stimulus types that differed only in there middle segments, indicating that the intervening portion of the stimulus either altered stored information about the onset portion or altered some aspect of the scaling judgment. For the stimuli in the Neuhoff (1998); Teghtsoonian et al. (2005), and all but the Static stimuli in the current Experiment 1, judgment of offset relative to onset (and offset relative to an initial plateau, as in Teghtsoonian et al., 2005) involved comparison across an intervening stimulus portion that differed in power from both the onset and the offset of the stimulus. It thus is possible that judgment of PSE for stimulus offset may depend upon whether the reference stimulus is presented prior to target stimulus onset (thus with the initial portions of the target stimulus intervening between the reference and targeted portion of the stimulus) or subsequent to the target stimulus offset (thus temporally adjacent to the targeted portion of the stimulus with only silence intervening). Because of these possibilities, PSE for offset magnitude should be evaluated relative to the reference stimulus presented prior as well as subsequent to the target stimulus.

A final important consideration in the design of Experiment 2 concerns the methodology used to measure PSE. When applied to difference limen or threshold (DL) measures, PSE is the midpoint of the interval of uncertainty (or equality) that defines the range between the values of the upper and lower DL. In principle, the PSE for loudness could be measured in the same way, generating the psychometric functions for detecting a difference in loudness, and thus loudness DL. However, neither the DL nor the midpoints of the upper-lower DL range (the classic "interval of uncertainty") is not highly relevant to the questions addressed in the current study. More important, although threshold-based PSE measures may become relevant in future studies, a potential problem with the threshold approach for the current study is that the listener judgment would no longer involve direct magnitude (ratio) scaling (as in Experiment 1), but rather an ordinal judgment of loudness, thus complicating interpretation of any results that might differ from those of Experiment 1. For this reason, PSE here is evaluated using ratio scaling of relative loudness between a reference standard and the specified portion of the target stimulus.

It is doubtful that Experiment 2, or any single experiment, can provide a complete understanding of the factors that are either similar or different for loudness perception across static and dynamic stimuli. The realistic goal of Experiment 2 
is to use the magnitudes of PSE values, and the similarities and differences across the Static and Dynamic stimuli to provide important insights about what factors are most probably relevant to loudness judgment and why.

\section{Methods}

\section{Participants}

There were a total of 148 participants ( 83 for the Static stimulus set, 65 for the Dynamic stimulus set) in Experiment 2. Participants were dropped from the study based upon whether their data met the a priori criteria described below. A total of 41 participants were dropped from the Static stimulus evaluations, leaving 14 participants in each of three conditions. A total of 20 participants were dropped from the Dynamic stimulus evaluations, leaving 15 participants in each of three conditions. For both types of stimuli, the majority of participants were dropped from the condition in which loudness at target stimulus offset was judged relative to the reference standard presented before the target stimulus onset.

\section{Stimuli}

Visual The circle stimuli from Experiment 1 were used to familiarize participants with the scaling task. On each trial, three circles were presented simultaneously on the monitor. Whether the auditory task for the participant was to judge the temporally adjacent or temporally remote portion of the target stimulus determined which of the circles served as the standard (assigned a reference value of 100) and which served as the comparison (relative magnitude to be judged; see below). Because reasonable PSE values can be estimated only if the psychometric functions are reasonable, the a priori criteria for inclusion (described below) are based upon the auditory data. The visual task thus was used solely to acquaint participants with the scaling procedure.

Auditory The target stimuli were the Experiment 1 Static and Dynamic stimuli with the $15-\mathrm{dB}$ change in power across the full $60-90 \mathrm{~dB}(\mathrm{C})$ range. Standard (or reference) stimuli were a set of four stationary, $600 \mathrm{~ms}, 1,000 \mathrm{~Hz}$ tones that ranged in amplitude $(-5,0,5$, and $10 \mathrm{~dB})$ around the actual or nominal power (Point of Objective Equality or POE) of the portion of the target stimulus (onset or offset) being evaluated. As an example, when evaluating the initial loudness of the $65-80 \mathrm{~dB}(\mathrm{C})$ target stimulus, the possible reference tones were $60,65,70$ and $75 \mathrm{~dB}(\mathrm{C})$. For the participant group that estimated loudness PSE for target stimulus onset, the reference stimulus on each trial always preceded the target stimulus (onset references). Loudness PSE for target stimulus offset was evaluated by two other groups of participants, one with onset references, and the other with the reference stimulus subsequent to the target stimulus (offset references) ${ }^{5}$. Whether the reference stimulus was at onset or offset, the ISI between the reference and target (Dynamic or Static) stimulus was always $600 \mathrm{~ms}$.

\section{Procedure}

Figure 5 summarizes the three different scaling conditions, but only for the Dynamic stimuli with increasing power. The conditions for the Dynamic stimuli with decreasing power would be illustrated by flipping the figure around the middle value of the ordinate. The analogous illustration for the Static stimuli would replace the Dynamic stimulus in this Figure (also illustrated in Fig. 1a) with the Static stimulus (illustrated in Fig. 1b). In the conditions corresponding to Fig. 5a, loudness of the onset of the stimulus is scaled relative to the loudness of the static reference stimulus presented prior to the onset of the target. In the other two panels, the loudness comparison is between the offset magnitude of the target stimulus and the magnitude of the static reference presented on the given trial, with the reference location either before the target (Fig. 5b) or after the target (Fig. 5c). For the conditions illustrated in Fig. $5 \mathrm{c}$, there is a 600 -ms silent interval between the offset of the target and the onset of the reference stimulus. For the conditions illustrated in Fig. 5b, there is a 2,400-ms interval between reference stimulus offset and the offset of the target stimulus, with that interval consisting of $600 \mathrm{~ms}$ of silence followed by the full 1,800ms duration of the target stimulus. Thus, the conditions illustrated in Fig. $5 \mathrm{~b}$ required storage of information about reference stimulus magnitude over a considerably longer period of time and over an intervening power magnitude that differed from both the reference stimulus and the offset of the target stimulus.

For the initial circle judgment task, three circles (left, middle, and right) were always presented simultaneously. The circle judgment task is analogous to the Static stimulus versions of the conditions illustrated in Fig. 5, but with spatial location substituted for temporal location. For the circle analogue to Fig. 5a, the left and (adjacent) middle circles are the reference and target stimuli respectively. For the circle analogue to Fig. 5b, the left circle again is the reference stimulus, but the right circle is the target stimulus. Finally, for the analogue to Fig. 5c, the middle circle and right circles serve as the target and reference stimuli. For both the visual and auditory task, the reference stimulus, although differing

\footnotetext{
${ }^{5}$ In the original design of Experiment 2, onset and offset PSE values were estimated using only onset references. The offset PSE condition for both stimulus types seemed to have been very difficult for the participants, resulting in the large number who failed to meet the inclusion criteria. Thus, the offset PSE values were for a new group of participants with references now at offset.
} 
Judge Onset of Stimulus

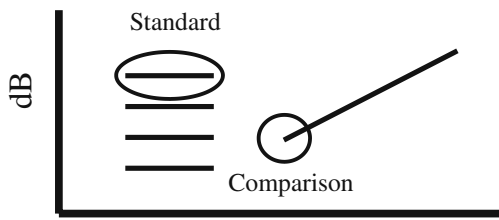

Judge Offset of Stimulus

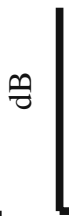

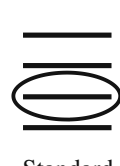

Standard

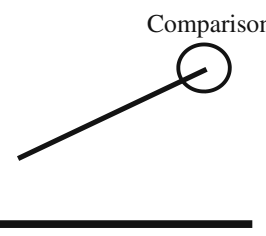

Time in ms
Judge Offset of Stimulus

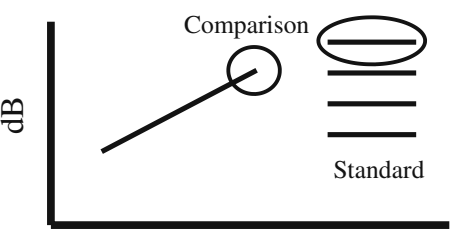

Fig. 5 Illustration of the types of auditory stimuli used in Experiment 2, but only for the Dynamic stimuli with increasing power. a Judgments of onset loudness, b judgments of offset loudness with reference at onset, and $\mathbf{c}$ judgments of offset loudness with reference at offset. Flipping each panel around the horizontal axis would illustrate the decreasing power

randomly in physical magnitude across trials, was always assigned a value of 100 , with the participant then producing a number that reflected the judged magnitude of the designated portion of the target stimulus relative to the reference.

There were six groups of participants: for the two (Static and Dynamic) types of auditory stimuli, there was a separate groups of participants for each of the stimulus conditions (each of the three panels in Fig. 5a, b and c), with each group first running the analogous circle condition. As with Experiment 1, each participant judged separate blocks of increasing and decreasing power, with half of the participants in each group first running the block of increasing power stimuli. The four separate $15-\mathrm{dB}$ onsetto-offset stimulus ranges from Experiment 1 (Table 1) were used. For each range, there were four trials for each reference stimulus magnitude, and thus a total of 64 trials within each (increasing or decreasing power) block of trials.

For the two types of auditory stimuli, psychophysical functions were computed for each of $\mathrm{dB}$ range (Table 1) and direction of power change using linear regression of the logarithm of the magnitude ratio of the target against the four $\mathrm{dB}$ values of reference stimuli. Each listener thus generated a total of eight psychophysical functions (four target stimulus $\mathrm{dB}$ magnitudes for each direction of power change). PSE values reflect the estimated reference stimulus magnitude that produced a target loudness rating of 100 . Because meaningful PSE estimates require reasonable psychophysical functions, the regression equation properties were also used to define three very stringent a priori criteria for inclusion of the participant in Experiment $2^{6}$. All

\footnotetext{
${ }^{6}$ The task in Experiment 1 was to rate the loudness of the onset and offset of a single stimulus, with approximately $20 \%$ of participants dropped because the circle scaling task indicated a failure to understand the scaling task. In Experiment 2 the same scaling task is further complicated by the presence of part of the target stimulus which may not be possible to ignore without considerable effort. The strict criteria in Experiment 2 eliminated data from not only those participants who failed to understand the task, but also those participants who were either unwilling to or unable to consistently cope with the more difficult task.
}

versions of the Dynamic stimuli. For the Static stimuli, replace the Dynamic stimulus in each panel with the equivalent Static stimulus (see Fig. 1). On each trial, one of the four $600-\mathrm{ms}$ reference stimuli is presented with a $600-\mathrm{ms}$ silent ISI between it and the 1,800-ms target stimulus

of a participant's data was removed from the study if any one of the following was exhibited in the regression equation for any one of their eight psychometric functions: (1) an $r^{2}$ value $<.50$, (2) regression equation slope $<.05$ ), or (3) aberrant (e.g., negative) regression equation intercept. Based upon these criteria, the majority of removed participants had judged offset loudness relative to standards at onset (illustrated in Fig. 5b) and, in the discussion below, this is taken as an indicator of the relative task difficulty for this condition.

\section{Results and discussion}

With highly similar PSE results across order (block of increasing versus decreasing power), the data for each direction of power change were pooled across block order. The average Loudness PSE values are plotted in Fig. 6, with the Static and Dynamic stimuli results respectively in the upper and lower rows of panels, and the increasing and decreasing power stimuli respectively in the left and right panels. The heavy solid diagonal line indicates equivalence of the PSE and the actual (or nominal) power of the target stimulus (Point of Objective Equality or POE), thus providing points of reference. The ordering of values on the abscissas for the decreasing power is reversed, with the higher power to the left. Thus, the POE line also indicates the direction of power change for the target stimuli. With onset references (i.e., Fig. 5a and b), PSE was estimated for both target stimulus onset and offset, and these data are plotted using circle symbols. To help distinguish between PSE for target onsets and offsets (a potential difficulty with the Static stimulus results), the circle symbols indicating target stimulus onsets have a gray fill (and always are on the left side of each panel). PSE for target stimulus offset also was estimated with offset references, and these data plotted as diamonds. To relate the PSE values for individual stimuli, the data point for each onset PSE (gray filled circle) is connected to the offset PSE value evaluated with onset references (solid line with open circle symbol) or offset 
references (broken line with diamond symbol). The slope of these lines indicates the perceived change in magnitude across that stimulus. The importance of reference stimulus location is indicated by the degree to which these lines can be distinguished from each other. The standard error at each data point is plotted, but often is too small to be seen.

\section{Static stimuli}

There are clear systematic trends in the PSE results, but also a few trend discrepancies to note first. In intensity judgment tasks, the end points of the range of intensities have sometimes been found to serve as anchors (e.g., Braida \& Durlach, 1972; Teghtsoonian, 1973), and there may be similar anchor effects in the current results. With the decreasing power stimuli (top right panel in Fig. 6), PSE matches POE for the highest power onset $(90 \mathrm{~dB})$ and lowest power offset $(60 \mathrm{~dB})$ when the reference stimulus is temporally adjacent. With the increasing power stimuli, the same PSE-POE equivalence is seen only for the highest power offset, and then only for onset reference stimuli. Except for these three PSE values (where PSE $=$ POE), the PSE data exhibit a remarkable consistency.

There are six sets of PSE values: each of the two directions of power change has one set of onset values and two sets of offset values that differ in the reference stimulus location. For every data set, the regression of PSE against POE resulted in a slope that approximates unity (0.98-1.11), with all $r^{2}$ values greater than 0.98 , indicating that PSE maps directly on POE, but with, as is evident in Fig. 6, a systematic bias for PSE to underestimate POE. A bias toward underestimation of POE has also been seen in the classic magnitude scaling literature (e.g., Woodworth \& Schlosberg, 1958). For the increasing power stimuli (upper left panel), the average PSE-POE difference at onset is $4.6 \mathrm{~dB}$, and is $3.2-4 \mathrm{~dB}$ for offset (depending on reference stimulus location). For the decreasing power stimuli (upper right panel), the average onset PSE-POE difference is $2.9 \mathrm{~dB}$, whereas the offset difference is only $1.8 \mathrm{~dB}$ with temporally adjacent (offset) references, but $5.8 \mathrm{~dB}$ with onset references. Thus, for the Static stimuli, there is an average tendency to underestimate magnitude by roughly $4 \mathrm{~dB}$.

In principle, the difference in mean PSE values between onset and offset estimates the change in effective subjective power across the target stimulus. These estimates are indicated graphically by the slopes of the lines in Fig. 6 that connect the onset and offset PSE values, with these most of these lines roughly parallel to the PSE $=$ POE line. The actual PSE values and resulting effective power change magnitudes are summarized for the Static stimuli in the upper portion of Table 2 . Using the $60-75 \mathrm{~dB}(\mathrm{C})$ results (upper row) to illustrate the components in this Table, the PSE for onset loudness (onset references) is $55.8 \mathrm{~dB}(\mathrm{C})$, with the PSE values for offset being 72.2 and $70.5 \mathrm{~dB}(\mathrm{C})$ when measured, respectively, with onset and offset references. With these PSE values, the effective change in power is $16.4 \mathrm{~dB}$ with onset references $(72.2-55.8)$ and $14.7 \mathrm{~dB}$ with temporally adjacent references (70.5-55.8). Overall, except for the highest (75-90) dB range, the increasing power stimuli tend to exhibit a small overestimate of power change. For the decreasing power stimuli, power change is overestimated when evaluated with the onset references, but when evaluated with the temporally adjacent references, there is a trend from underestimating power change at the lowest (75-60) power range to overestimating power change at the highest $(90-75)$ power range. Thus, judgment of stimulus magnitude is altered when a different power level intervenes between the stimuli or stimulus components being compared both within stimuli (Hybrid vs Static stimuli in Experiment 1) and across stimuli (Static stimuli in Experiment 2). Overall, other than possibly some of context (intervening power level) and anchor effects, the Experiment 2 results for the Static stimuli are fairly orderly, but do not provide a simple basis for understanding the Experiment 1 results.

\section{Dynamic stimuli}

The PSE values for the Dynamic stimuli are summarized in the bottom part of Table 2 and displayed graphically in the lower panels of Fig. 6. Auditory system temporal integration should result in PSE values that are regressed somewhat toward the median stimulus power, which should result in smaller within stimulus changes in effective power. These differences from the nominal physical specifications of the stimuli should be seen graphically in Fig. 6 (bottom panels) as shallower slopes within stimulus lines, with the higher power POE data points below and the lower power POE data points above the PSE $=$ POE diagonal. This general pattern of data is quite evident in Fig. 6 (bottom panels), with not only this pattern, but the absence of a systematic (i.e., $4 \mathrm{~dB}$ ) underestimation bias, representing clear differences relative to the Static stimulus results (upper panel). Although the general pattern of Dynamic stimulus results is consistent with the basic notion that temporal integration truncates the effective power range, the slopes of these within stimulus lines are considerably shallower than predicted by simple temporal integration.

Assuming perfect integration of power, the effective power of the onset and offset of a Dynamic stimuli should differ from the nominal $\mathrm{dB}$ power by between 0.42 and $1.25 \mathrm{~dB}$, with these values reflecting the minimum $(100 \mathrm{~ms})$ and the maximum possible $(300 \mathrm{~ms})$ integration time. The maximum value $(1.25 \mathrm{~dB}$ for $300-\mathrm{ms}$ integration window) will be used to represent the largest change in effective power due to temporal integration. If temporal 
Fig. 6 Average PSE values for Static (upper row) and Dynamic (lower row) stimuli of increasing power (left column) and decreasing power (right column). The heavy solid diagonal line indicates equivalence of the PSE and the actual (or nominal) power of the target stimulus (Point of Objective Equality or POE). Onset PSE values are represented by gray filled circles. Offset PSE values estimated from standards at onset and offset are represented by open circles and diamond symbols respectively
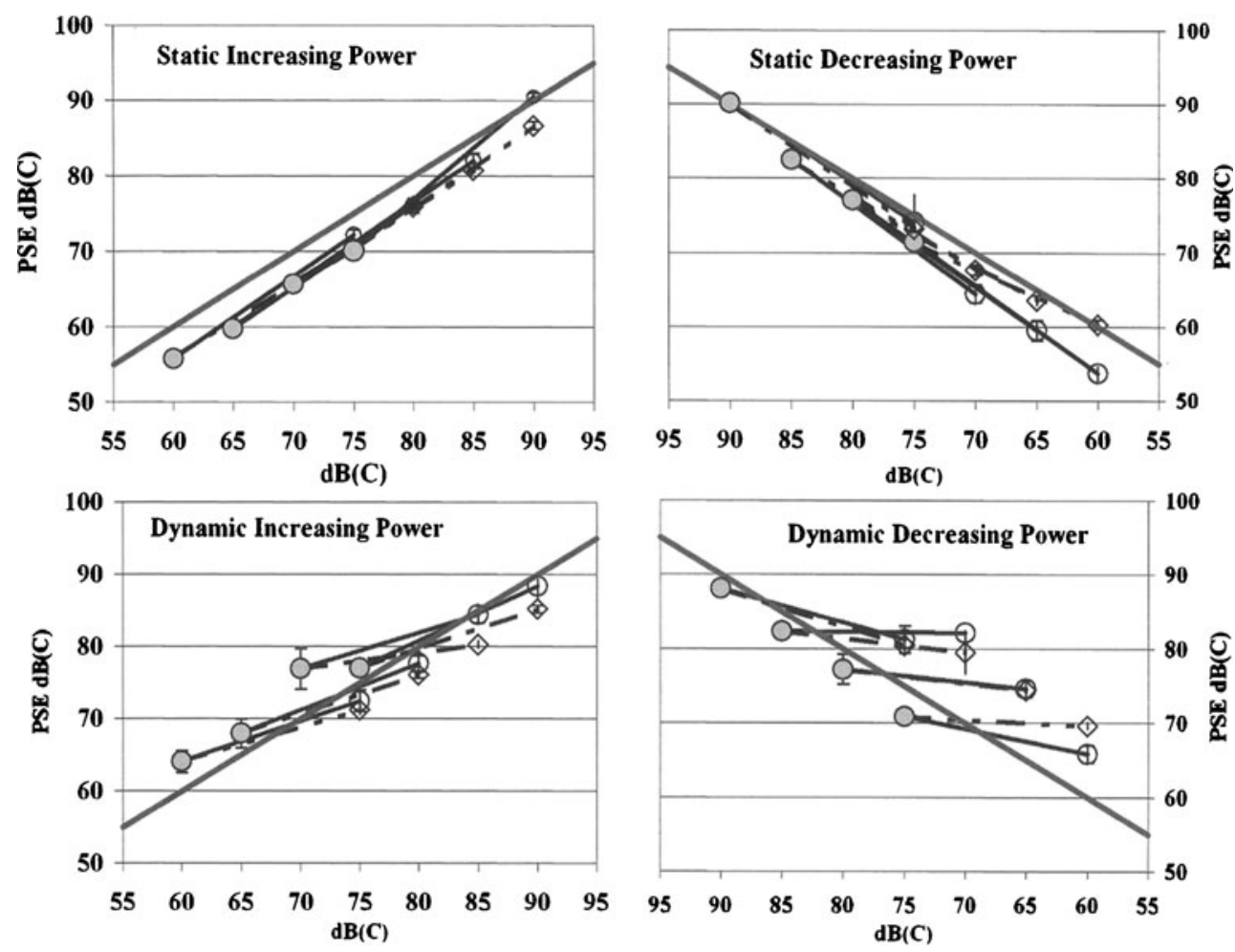

integration were the only difference between the Dynamic and Static stimuli, then $|\mathrm{POE}-\mathrm{PSE}|$ should always be approximately $1.25 \mathrm{~dB}$, with direction of difference $( \pm)$ reflecting regression toward the median stimulus power. For the increasing power Dynamic stimuli, the average |POE-PSE| value is $4.0 \mathrm{~dB}$ for stimulus onset and either 1.9 or $4.3 \mathrm{~dB}$ for offset, depending upon the location of the reference stimuli. For the decreasing power Dynamic stimuli, the average |POE-PSE| value is 2.9 for stimulus onset and approximately $8.5 \mathrm{~dB}$ for stimulus offset with either reference stimulus location, with all of these POEPSE $\mid$ reflecting regression toward median power. The |POEPSE $\mid$ differences clearly are greater than the maximum $1.25 \mathrm{~dB}$ expected simply from temporal integration.

If the auditory system's temporal integration process is leaky, effective power would be shifted to more heavily weight the latter in that portion of the stimulus located within any temporally-defined integration window. This possible change in integration weighting should be of little consequence for the stationary initial and final components of the Static stimuli. For the Dynamic stimuli, this possible change in integration weighting might shift effective power in the direction of the power change, and thus toward the ending, rather than median, power of the stimulus within any integration window, resulting in larger |POE-PSE $\mid$ values for stimulus onset than offsets. Since the largest |POE-PSE $\mid$ values are for the offsets of the decreasing power stimuli, this possibility is not reasonable.
For the Dynamic stimuli, the initial and final PSE values translate into reduced magnitudes of effective within stimulus power changes (Table 2, bottom panels). The size of the differences across Static and Dynamic stimuli is too large to be explained solely by temporal integration. If the only difference across stimulus type was due to simple temporal integration, the effective change in power should be lower for the Dynamic stimuli by a consistent maximum of $2.5 \mathrm{~dB}^{7}$ (i.e., a maximum of $1.25 \mathrm{~dB}$ for both onset and offset). For the Dynamic stimuli, the average estimated effective power change is not a minimum of $12.5 \mathrm{~dB}$, but rather less than $10 \mathrm{~dB}$ for the increasing power stimuli and an average of only $3.7 \mathrm{~dB}$ for decreasing power. Although the effective change magnitudes are consistently greater for the increasing power version of these stimuli, and thus exhibit ordinal consistency with the Neuhoff (1998) loudness change findings, the magnitude of the difference is probably not. Specifically, although a comparison between ratio and interval data is problematic, a roughly $6-\mathrm{dB}$ difference (e.g., 3.7-10 dB) seems much too large to assume magnitude consistency with the Neuhoff data. This same roughly 6-dB difference across direction of power change also seems too large to provide a simple basis for understanding the Experiment 1 magnitudes of loudness change either for the

\footnotetext{
${ }^{7}$ Assuming that total power change across a stimulus reflects this $1.25 \mathrm{~dB}$ temporal integration difference between effective and nominal POE at both onset and offset, the expected effective power change is (as specified earlier) $2.5-\mathrm{dB}$ lower than the nominal $15-\mathrm{dB}$ power change for this stimulus.
} 
Table 2 Estimates of effective power change based upon Experiment 2 PSE results
Estimates of change in effective subjective power across target stimuli

\begin{tabular}{|c|c|c|c|c|c|c|}
\hline \multicolumn{2}{|c|}{ Power dB(C) } & \multirow{2}{*}{$\begin{array}{l}\text { Onset PSE } \\
\text { Onset Ref }\end{array}$} & \multirow{2}{*}{$\begin{array}{l}\text { Offset PSE } \\
\text { Onset Ref }\end{array}$} & \multirow{2}{*}{$\begin{array}{l}\text { Offset PSE } \\
\text { Offset Ref }\end{array}$} & \multicolumn{2}{|c|}{ Effective dB Change } \\
\hline Onset & Offset & & & & Onset & Adjacent \\
\hline \multicolumn{7}{|c|}{ Static Stimuli: Increasing Power } \\
\hline 60 & 75 & 55.8 & 72.2 & 70.5 & 16.4 & 14.7 \\
\hline 65 & 80 & 59.8 & 76.1 & 76.1 & 16.3 & 16.3 \\
\hline 70 & 85 & 65.7 & 82.0 & 80.8 & 16.3 & 15.1 \\
\hline \multirow[t]{2}{*}{75} & 90 & 70.1 & 90.5 & 86.7 & 20.4 & 16.6 \\
\hline & & & & Average & 17.4 & 15.7 \\
\hline \multicolumn{7}{|c|}{ Static Stimuli: Decreasing Power } \\
\hline 75 & 60 & 71.5 & 53.7 & 60.3 & 17.8 & 11.2 \\
\hline 80 & 65 & 77.1 & 59.5 & 63.6 & 17.6 & 13.5 \\
\hline 85 & 70 & 82.6 & 64.5 & 67.7 & 18.1 & 14.9 \\
\hline \multirow[t]{2}{*}{90} & 75 & 90.1 & 74.1 & 73.2 & 16.1 & 16.9 \\
\hline & & & & Average & 17.4 & 14.1 \\
\hline \multicolumn{7}{|c|}{ Dynamic Stimuli: Increasing Power } \\
\hline 60 & 75 & 64.0 & 72.5 & 71.2 & 8.4 & 7.2 \\
\hline 65 & 80 & 67.9 & 77.6 & 76.1 & 9.7 & 8.2 \\
\hline 70 & 85 & 76.9 & 84.4 & 80.3 & 7.5 & 3.4 \\
\hline \multirow[t]{2}{*}{75} & 90 & 77.0 & 88.4 & 85.2 & 11.4 & 8.2 \\
\hline & & & & Average & 9.3 & 8.0 \\
\hline \multicolumn{7}{|c|}{ Dynamic Stimuli: Decreasing Power } \\
\hline 75 & 60 & 70.9 & 65.8 & 69.7 & 5.0 & 1.2 \\
\hline 80 & 65 & 77.2 & 74.6 & 74.4 & 2.6 & 2.8 \\
\hline 85 & 70 & 82.3 & 82.1 & 79.5 & 0.3 & 2.8 \\
\hline \multirow[t]{2}{*}{90} & 75 & 88.2 & 81.2 & 80.4 & 6.9 & 7.7 \\
\hline & & & & Average & 3.7 & 3.7 \\
\hline
\end{tabular}

Dynamic stimuli across direction of power change or across the Static and Dynamic stimuli for each direction of power change (see Fig. 3, middle panels). Although, the differences in PSE results for the Static and Dynamic stimuli do not provide a simple basis for understanding the qualitative similarities and quantitative differences across stimulus type in the Experiment 1 results, they do provide the basis for some important insights into similarities and differences in loudness perception for static and dynamic stimuli.

\section{General discussion}

What is being investigated?

Before turning to the implications of the current study, there is the important question of what was, and was not, being investigated in the various studies of loudness for dynamic stimuli. Neuhoff (1999) provided one crisp contrast between the nature of his loudness investigation of dynamic stimuli, the Canévet et al. study of equivalent dynamic stimuli, and classic studies of loudness for static stimuli. According to this contrast, the Neuhoff listeners "were specifically asked to ignore the overall loudness of sounds and to make summary judgments about the amount of loudness change ..." whereas the other studies evaluated "a discrete measure of loudness at various snapshots in time" "assigning a number to the loudness ... at (those) various times ..." (Neuhoff, 1999, p. 673). Although a sharp contrast can be useful, there is a major problem with this one. The only measure that reflects any aspect of overall loudness is the very old, seldom used, largely historical "Phon" scale. The Phon is a measure of the physical magnitude (dB:SPL) of a $1,000-\mathrm{Hz}$ tone that is judged to be equal in loudness (1:1 loudness ratio) to the stimulus being evaluated. The Phon scale provides only ordinal information about loudness (e.g., a 92- and a 12-Phon stimuli are both louder than an 11-Phon stimulus). Loudness, if a "prothetic" continuum, can only be measured meaningfully on a ratio scale, which requires comparing two physical stimuli (e.g., Stevens, 1961). For example, the "Sone" scale reflects the loudness ratio between the pair of compared stimuli; stimuli that are separated by 2 Sones (or $1 / 2$ Sone) have a subjective loudness ratio of $2: 1$ (or 1:2), independent of the overall physical power, difference in power, and spectral composition of the stimuli. However, most tradi- 
tional psychophysical studies do not bother with the "Sone" label, and simply evaluate the loudness ratio between a pair of acoustic stimuli or stimulus elements.

Although the exact wording of instructions may differ across the studies cited by Neuhoff (1999), all the studies were evaluating loudness difference or change between a pair of stimuli or stimulus elements presented to the listener; none were measuring overall or absolute loudness of the stimuli at any single point in time. ${ }^{8}$ Ratio scaling can be used (the traditional literature argues should be used) to evaluate any pair of stimuli or stimulus elements in any stimulus context, whether the stimuli are stationary or dynamic, continuous or discrete, and across any degree of temporal or spatial separation. The major difference across studies is not the basic question being asked, but rather that Neuhoff used interval, rather than ratio, scaling to evaluate the difference or change across the compared stimulus components or elements. A separate and long debated issue is whether Stevens' power law is more than a sometimes useful heuristic, but that debate is not critical to the current discussion. The immediate important questions concern (1) whether or not there are specific systematic differences in the (proportional) change in loudness as a function of the stimulus attributes investigated, and (2) if there are differences, the probably cause of those differences. The current study addresses these questions for static versus dynamic stimuli, with the results indicating primarily quantitative, rather than qualitative, differences.

\section{Implications from current study}

The first goal for the current study was to determine whether there are differences in loudness perception for stationary (static) and dynamic changes in power for the $1,000-\mathrm{Hz}$ tone stimuli investigated in recent studies (i.e., Neuhoff, 1998; Teghtsoonian et al., 2005). In Experiment 1, the direct scaling of the offset to onset loudness ratio resulted in the same pattern of loudness-power relationship across all four stimulus types investigated, directly challenging the assertion "that there are dramatic differences between static and dynamic loudness perception" (Neuhoff, 1998, p. 123). Although there are quantitative differences in the loudness across stimulus type, these are probably due largely to at least two factors. First, because of temporal integration by the auditory system, the effective power of the initial and final portions of the Dynamic stimuli in the current, as well as in the Neuhoff $(1998,2001)$ and

\footnotetext{
${ }^{8}$ In Experiment 2, the PSE values indicate physical magnitude that, based upon ratio scaling, resulted in loudness equivalence for the broadly defined onset or offset portions of the target stimulus. Rather than representing absolute loudness judged at a specific point in time, this represents a 1:1 ratio for what the listener judges to be the onset and portions of the target stimulus.
}

Teghtsoonian (2005) studies, differs from the nominal power, meaning that the effective change in power for these stimuli differs from static (stationary) stimuli with the same initial and final power labels. This aspect of the static and dynamic loudness perception reflects known physical and psychophysical properties of sound. Although temporal integration, as discussed above, clearly does not account for the magnitude of the quantitative differences, another aspect of temporal integration (discussed below) may contribute to the magnitude of difference found in the two experiments.

A second factor in the differences across stimulus type is probably some form of contextual effect (e.g., Backward Recognition Masking; Pastore, Gaston, \& Berens, 2008). Based upon the incidence of failure to meet the inclusion criteria in Experiment 2, it is clear that participants found it most difficult to judge loudness of stimulus offset when the reference was presented prior to target stimulus onset, and thus with the initial portion of the target stimulus intervening between the stimulus elements being contrasted. The actual PSE results also indicate a difference in judged magnitude when there is an intermediate level of power between the stimuli (or stimulus components) being compared. The stimuli in the Neuhoff (1998) and Teghtsoonian et al. (2005) studies, and all but the Static stimuli in the current Experiment 1, resulted in loudness judgments between final and initial portions of the target stimulus separated by the identical stimulus at an intermediate power level. The Experiment 1 results also indicate that, beyond an effect of an intervening intermediate power level, the nature of that intermediate component seems to be important. This was demonstrated both by the differences in results across the two types of Hybrid stimuli, as well as the separate quantitative grouping of the results for the Static and Hybrid-Static versus the Dynamic and Hybrid-Dynamic stimuli (Fig. 3). Although the importance of this latter dynamic factor might be claimed to represent a difference in the processing of loudness for dynamic versus static stimuli, it does not conform to the static-dynamic contrast claimed by Neuhoff (1998), as the intervening stimulus element in HybridDynamic stimulus is providing a context in which the evaluated stimulus elements are presented and, at least in principle, is not itself being directly evaluated. Furthermore, this basic notion that contextual stimuli, or stimulus elements, can alter perception or perceptual judgment is certainly not new (e.g., masking, adaptation level effects, range effects, or anchor effects).

Temporal integration and temporal uncertainty

A possible factor that may contribute to quantitative differences in loudness judgment of static and dynamic stimuli is temporal uncertainty. Temporal uncertainty may 
be a by-product of the temporal integration aspect of stimulus processing by the auditory system. Consider two different, relatively extreme models of a process that integrates power across a fixed duration (e.g., a 300-ms integration window is used solely to illustrate the models). The integration process in each model adds the next $n$ ms of power while simultaneously deleting the oldest $n$ ms of power. The output of both models, which reflects integrated stimulus magnitude, then is used by the later loudness judgment processes. The first, Gated model, is essentially ideal. It has extra information that allows it to be synchronized with stimulus onset and offset. This not only gives it the ability to only operate when a stimulus is present but also allows it to easily identify the integrated power that corresponds to stimulus onset and offset. There thus is no temporal uncertainty about what constitutes power magnitude at stimulus onset and offset. This model is the basis for the predicted maximum 1.25-dB difference in onset and offset power between the Dynamic and actual $15-\mathrm{dB}$ change in the other three types of stimuli investigated in this study.

The alternative model considered, the Free-Running model, operates continually, whether or not the stimulus is present. Rather than having added information that precisely specifies stimulus onset and offset, that information must be estimated based upon the output of the integrator. This means that there is temporal uncertainty about what represents power at onset and offset, and both the degree and importance of this temporal uncertainty should differ for the Static and the Dynamic stimuli. Consider the output of the $n \mathrm{~ms}$ integration window $(100<n<300)$ as a function of its temporal location relative to the ongoing physical stimulus. The integrator output near onset and offset depends not only on the instantaneous power of the physical stimulus, but also upon the portion of the integration window filled (and not filled) by the stimulus. For each of the two (600 ms initial and final) stationary components to the Static type stimuli (e.g., Fig. 1a, b and c), starting at the physical onset of the stimulus, integrated power will increase systematically until the integration window is being filled (e.g., over the initial $n \mathrm{~ms}$ ), and then is constant for an extended period of time (e.g., $600-$ $n \mathrm{~ms})$. Beginning at the offset of the physical stimulus, this period of constant effective power is followed by a period of decreasing power as the integration window extends increasingly beyond the end of the physical stimulus. There thus are differences in the rate of change in integrator output that are associated with physical stimulus onset and offset. Not only is there at least moderate information to help identify the onset (and offset) of the stimulus (and thus low temporal uncertainty), but because there is an extended period of time (when the integrator window fully overlaps in time with the physical stimulus), there is no variability in output over this period of time. Therefore, the loudness judgment of change across the stimulus should be both relatively accurate about what constitutes the initial and final power of the stimulus and also should be highly tolerant of considerable temporal uncertainty in identifying stimulus onset and offset.

In contrast, there is considerable temporal uncertainty and little tolerance for such uncertainty when evaluating loudness change for the Dynamic stimuli. For the Dynamic stimuli with increasing power, the output of the FreeRunning Integrator model initially will increase rapidly, but the rate of increase will decelerate until a constant rate of increase is achieved (i.e., once the stimulus first completely fills the integration window). The integrated power then will continue to change, now driven solely by, and thus reflecting, the change in physical power. Once the physical stimulus ends, the integration output will begin decreasing rapidly as contribution of past stimulus power systematically decreases. There thus is quite imprecise information about the integrated power that represents the initial and terminal aspects of the stimulus. Although beyond the scope of the current discussion, there may be differences across the direction of power change for the Dynamic stimuli (e.g., at onset, once the integration window is filled, the integrated power will slowly adjust to the direction of change in physical power). The important points are that, because the integrated power is continually changing, (1) the evaluated offset/onset ratio depends upon the temporal locations of integrator chosen to represent onset and offset, and (2) this continual change in integrated power across time may make it difficult for the listener to determine the integrated output information that actually represents the onset and offset of the stimulus. If loudness change is the ratio of the estimated power at stimulus offset relative to stimulus onset, then the judgments for dynamic stimuli should be dependent upon the nature and degree of temporal uncertainty, with the possible range of uncertainty always skewed toward the temporal center of the physical stimulus.

Although the two models described are probably extreme, as long as the actual human auditory system integration process is even only somewhat more like the Free-Running model than the ideal Gated model, then, in contrast to the Static stimuli, when loudness judgment is made for Dynamic stimuli, there will be both high temporal uncertainty about what is to be judged and little tolerance for such temporal uncertainty. There thus is a theoretical basis for the notion that temporal uncertainty may be a factor in loudness perception of dynamic stimuli, and this may provide, or at least contribute to, an explanation for the contrast in results for the Dynamic stimuli across the two current experiments. In Experiment 1, temporal uncertainty should be a factor in the judgment of both contrasted 
stimulus components (i.e., the nominal onset and offset power). In Experiment 2, temporal uncertainty should be a factor for the evaluated component of Dynamic target stimulus, but not for the steady-state reference stimuli. The fact that different results were obtained in the two experiments for the Dynamic stimuli not only lends at least indirect support for the temporal uncertainty notion, but the power change direction difference in PSE results for these stimuli also may imply that the importance of temporal uncertainty may differ across direction of power change. Temporal uncertainty, however, would not provide a simple explanation for the Experiment 1 quantitative differences in loudness change across the two Hybrid type stimuli, where the onsets and offsets are identical $600 \mathrm{~ms}$ stationary components.

\section{Looming}

The Looming conjecture links change in power to sound source motion and then, because of the presumed evolutionary (predator-prey) importance of approaching sources, to cause in differences in loudness change magnitude. The idea that loudness judgments may reflect judgments of source-listener distance is not new (e.g., Warren, 1958, 1981), but also not widely accepted. Looming is a real phenomenon that has been studied empirically for some time (e.g., Ghazanfar, Neuhoff, \& Logothetis 2002; Rosenblum, Carello, \& Pastore 1987; Rosenblum, Wuestefeld, \& Saldaña, 1993), and is not in question. In addition, distance is probably an important factor in predator-prey interactions. Neuhoff (e.g. Neuhoff, 1998, 2001; Seifritz et al., 2002) has linked these concepts. In this evolutionary perspective, approaching objects (sound sources) are more important than stationary or receding objects, and this difference in importance is conjectured to lead to a bias towards underestimating time-to-arrival. This bias then is conjectured to lead to increased judgments of change in subjective magnitude for increasing power relative to stationary or decreasing power stimuli. The conjectured consequence is that the final portion of an increasing power stimulus is perceived as louder than the final portion of a decreasing power stimulus. It is specifically this linking conjecture that is in question, and this conjecture can be examined from several perspectives.

Because sound power at the location of the listener is inversely proportional to the square of the source distance, the linear change in $\mathrm{dB}$ across time for the dynamic stimuli studied would represent a source that is slowing down as it approaches (traveling half the previous distance for each 6- $\mathrm{dB}$ change in power) and speeding up as it recedes (traveling double the previous distance for each 6-dB change), which leads to the question about whether it is simply looming, or another aspect of the presumed source movement that is conjectured to be important to the listeners in the Neuhoff studies. If the critical factor is the argument that any change in power conveys to the listener a change in distance that will alter perceived change in loudness, then any change in power across time (including that in the four stimulus types in the current study) should exhibit greater loudness change for increases than decreases in power. A corollary to this is that, when there is any change in power, the subjective magnitude of a given physical power should depend upon both relative power and temporal order of each power change. As noted earlier, the Experiment 1 results (Fig. 4) indicate that for any power change that differs solely in temporal order, and thus direction (e.g., 60-75 vs 75-60), greater power change magnitude is not always found for increasing power, but rather depends systematically upon the nature of the stimulus and the overall magnitude (e.g., median) of the stimulus.

Experiment 2 provides direct estimates of PSE values, but for a more limited range of stimuli and power changes. The values for the Dynamic stimuli do indicate that the perceived magnitudes of change are smaller for decreasing than increasing power. This aspect of the current results would seem to be consistent with Neuhoff's findings and his conjecture, but this type of finding was the original basis for his looming conjecture. However, two aspects of the current results are not logically consistent with this conjecture. First, the looming argument ${ }^{9}$ should predict that, when the ending of an increasing power stimulus and the beginning of a decreasing power stimulus are identical in amplitude, the offset PSE values of the increasing power (looming) stimulus should be greater than the onset PSE values for decreasing power (receding) stimulus. In the current study, this power equivalence occurs only for the $90-75$ and $60-75 \mathrm{~dB}$ stimuli. For the Static stimuli (Table 2), the $75 \mathrm{~dB}(\mathrm{C})$ offset component has an average PSE of $73.7 \mathrm{~dB}$ when preceded by the more intense onset (ostensibly, the receding condition) and only $70.7 \mathrm{~dB}$ when preceded by the less intense onset (ostensibly looming condition). The pattern is the same, but more dramatic, for the Dynamic stimuli, with respective average PSE values of 80.8 and $71.6 \mathrm{~dB}$. At face value, these latter results would seem to be strong evidence against the looming conjecture. However, the Dynamic (but not the

\footnotetext{
${ }^{9}$ One could argue that the sudden appearance of a loud stimulus could be perceived as the source being close to the listener and thus, although not looming, has great importance, and thus is perceived as louder than it otherwise should be. However, such a post hoc conjecture should then predict an enhanced contrast between the onset and offset power of such stimuli, and thus possible equality to the conjectured looming induced enhancement.
} 
Static) PSE comparison is complicated by consideration of any simple notion of temporal integration that would result in greater effective power for the decreasing than increasing power stimuli. Still, this $9 \mathrm{~dB}$ difference is far greater than the maximum $2.5-\mathrm{dB}$ difference predicted from the Gated model of temporal integration described above.

A variation on the logic of this last analysis is a comparison of PSE values for the pairs of temporal reversal of the same stimuli (e.g., the $60-75$ and $75-60 \mathrm{~dB}(\mathrm{C})$ stimuli are identical, but temporally reversed). Not only are these stimuli physically identical in all respects except for temporal ordering, but the Gated model of temporal integration predicts identical effective power for the pair of temporally reversed Dynamic stimuli. The logic of the looming conjecture is that, for such identical physical changes, the version of the stimulus with power increasing across time should result in greater change in loudness. The comparison of Experiment 1 results summarized in Fig. 4 do demonstrate directional differences, but ones that depend systematically upon both stimulus type and median power, rather than the consistent bias claimed by the looming conjecture.

The importance of direction of power change for the Experiment 2 results also can be evaluated using a variant of the logic in the previous paragraph. PSE for every $\mathrm{dB}$ component value can be evaluated at the onset of an increasing power and the offset of a decreasing power stimulus. If looming is a significant factor in the Loudness-Power relationship, then the PSE values should always be greater at the offset of every increasing power (looming source) stimulus than at the onset of its temporally reversed, decreasing power (receding source) stimulus. Figure 7 summarizes the Experiment 2 PSE values for such pairs of stimuli at each (nominal) POE. The solid lines with open symbols are for increasing power, the broken lines and filled symbols are for decreasing power, and the triangles and squares respectively indicate target onset and offset. For the Static stimuli (upper panels), there are only small differences across location within the target stimulus. When the reference stimulus is at onset (left panel), there is a systematic ordered relationship, with higher PSE values for the nominally receding, decreasing power stimuli (filled symbols and broken lines) than the nominally looming, increasing power stimuli. When the reference stimuli are temporally adjacent to the component being judged (right panel), there is no systematic ordering. Thus, the Static stimuli do not exhibit the expected looming conjecture relationship.

For the Dynamic stimuli (lower panels), the pattern of findings are consistent across reference stimulus location (left versus right panels), with PSE values always higher for the nominally receding, decreasing power stimulus at the lower POE values ${ }^{10}$, but with little difference at the higher POE values. Thus, rather than support, these data represent, if anything, evidence against the looming conjecture. Although a post hoc argument can probably be made against this analysis of the looming conjecture, the essential question should not be whether the simple, single conjecture about looming is or is not valid, but rather what factors contribute to the power measurement and loudness scaling by the human auditory system across different types of stimuli, and clearly there are a number of important factors. For example, in this last analysis, there is a notable difference between the Static and Dynamic stimulus results. For the Static stimuli, PSE appears to be a single, continuous function of POE. For the Dynamic stimuli, the PSE-POE relationship is systematically different for the lower than the higher range of POE values.

Finally, although Neuhoff (1999, p. 673) refers to his "recent discovery of a bias for rising intensity tones", the finding of a difference between small-to-large and large-tosmall judgments is not a recent, unique finding. Poulton (1968) reports a number of studies (e.g., loudness, weight, reflectance) where, when using a relatively large modulus followed by judgment of a comparison stimulus, steeper slopes (larger exponents) are typically found for magnitude estimates above the standard than for below the standard. Poulton (1969) again reports this trend when comparing his investigation of loudness for stimuli above the standard with the Stevens and Poulton (1956) investigation of loudness for stimuli below the standard. This reported asymmetry with a single standard is similar to the roving standard loudness PSE results at the higher median stimulus magnitudes for all but the current Static stimuli (Fig. 7, except possibly at the $15-\mathrm{dB}$ change for the lowest stimulus range), as well as results in Teghtsoonian et al. (2005). If one assumes that the stimulus-initial magnitude is the standard for judging change, the Neuhoff (1998) pattern of results (although difficult to interpret because of the interval scaling) would be qualitatively consistent with the trend described by Poulton (1968). Of note, temporally-

\footnotetext{
${ }^{10}$ A common finding for judgments of loudness for sequential stimuli, and a conjecture by one reviewer as a possible contributing factor in the Experiment 1 results, is ILR or Induced Loudness Reduction (Epstein, 2007; Marks, 1988; Scharf, Buus, \& Nieder, 2002; Wagner \& Scharf, 2006). In typical demonstrations of ILR, a higher intensity tone preceding a target tone results in decreased loudness estimations of the target when the tones are separated by at least several hundred milliseconds (Marks, 1988). ILR develops fairly rapidly, declines slowly, and probably accumulates over trials (Epstein, 2007). However, an overall decrease in loudness estimations for decreasing as opposed to increasing power (predicted by ILR) is not observed in any of our experiments, nor in the experiments of Neuhoff (1998) or the Teghtsoonian et al. studies $(2000,2005)$. Therefore, analyses of ILR for this investigation are not conducted.
} 
Fig. 7 Comparison of PSE values for identical power magnitudes (POE) as a function of increasing (solid lines) versus decreasing (broken lines) power and target stimulus onset (squares) versus offset (triangles). Static and Dynamic stimuli are plotted in the upper and lower panels, respectively. Left and right panels plot for PSE for stimulus offset judged evaluated with reference stimuli located at, respectively, at onset and offset
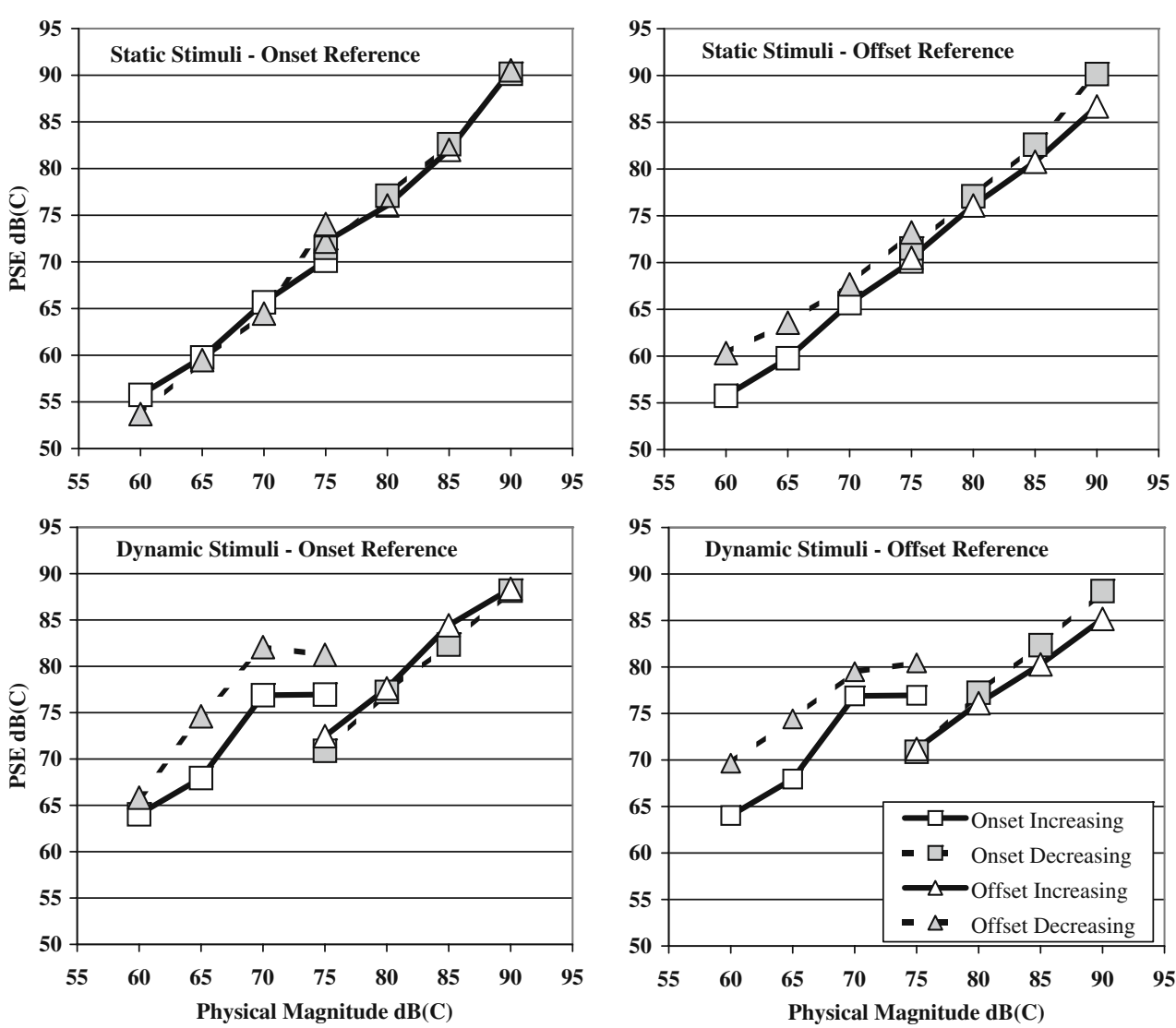

dependent judgments have also been demonstrated for estimations of duration (Grassi \& Darwin, 2006) and the sound quality of ramped and damped sinusoids (Irino \& Patterson, 1996). However, it is doubtful that the directional differences identified in the recent literature are simply attributable to the type of finding described by Poulton. It is more likely that these results reflect a combination of sensory judgment, information storage, perceptual interaction, and decision bias factors, possibly with other factors yet to be identified, and nearly all needing empirical investigation.

\section{A final comment on methodology}

Canévet et al. (1999, p. 673) correctly warn, "Close attention should also be paid to procedural differences that may turn out to be decisive." The current study only investigated a specific set of stimuli, and the recent studies by the Teghtsoonians, Canévet, Scharf, and others (cited above) demonstrate limits on the generalization of findings to other power ranges and durations. Because many of the cited investigations do differ in methodology and exhibit somewhat different patterns of results, it is not easy to draw broad conclusions across studies, and broad conclusions may not even be warranted. For example, the nature of the representations of both power and loudness, and such fundamental questions as whether scaling results actually reflect internal judgments of magnitude or reflect something more about the use of numbers, have been long and extensively debated (e.g. Gibson, 1966; Neuhoff, 1998, 2001; Warren, 1981; Zwislocki \& Goodman, 1980), and it is not clear that we are closer to a definitive resolution. Given the complexity of both the basic underlying principles and the various recent findings, conclusions about differences in loudness judgments between discrete stationary and dynamic stimuli should not be, and are not, simple. Undoubtedly, the method used to evaluate listener perception of loudness influences the quantitative results (e.g. Canévet et al., 1983; Poulton, 1968, 1969; Zwislocki \& Goodman, 1980; current Experiments 1 and 2). However, the equivalence in the qualitative patterns of current Experiment 1 results across stimulus type seems to indicate at least some level of commonality of underlying processes, and this would not be consistent with the conjecture that there are separate and highly distinctive processes for discrete stationary and dynamic stimulus changes. Although temporal integration was expected to produce quantitative differences across stimulus types, it seems inadequate to account for either the magnitude of those differences or the differences across the two current experiments. Temporal uncertainty, a logical consequence of temporal integration, should interact with both stimulus 
type and the methods employed in the two experiments, but certainly does not provide anything even starting to approach a complete understanding of loudness perception. The differences in loudness for dynamic power change across different durations in the several studies by Cavenet and the Teghtsoonians (summarized above) certainly indicates that loudness perception is a function of stimulus duration. Rather than focusing on a single conjecture about dynamic loudness perception, a careful, broad perspective is needed. It is only the systematic empirical investigation of the nature of qualitative and quantitative similarities and differences across stimulus type, power magnitude, context, and methodology that can ultimately provide a better understanding of the stimulus-perception (psychophysical) relationship. The current study is just one step in this important effort.

Acknowledgments The authors thank Jeremy R. Gaston for his comments and suggestions on both earlier and the final draft of the manuscript. They also thank John Neuhoff and especially Robert Teghtsoonian for their extremely useful comments and suggestions on earlier drafts of this manuscript. This material is based upon work supported by the National Science Foundation under Grant No. 0213666. Any opinions, findings, and conclusions or recommendations expressed in this material are those of the authors and do not necessarily reflect the views of the National Science Foundation.

\section{References}

Braida, L. D., \& Durnach, N. I. (1972). Intensity perception. II. Resolutions in one-interval paradigms. Journal of the Acoustic Society of America, 51(2), 483-502.

Canévet, G. (1986). Estimation de sonie pour de sons purs à variation monotone de niveau. Acustica, 62, 2136-2142.

Canévet, G., \& Scharf, B. (1990). The loudness of sounds that increase and decrease continuously in level. Journal of the Acoustic Society of America, 88(5), 2126-2142.

Canévet, G., Scharf, B., \& Botte, M.-C. (1983). Loudness adaptation, when induced, is real. British Journal Of Audiology, 17(1), 4957.

Canévet, G., Scharf, B., Schlauch, R. S., Teghtsoonian, M., \& Teghtsoonian, R. (1999). Perception of changes in loudness. Nature, 398, 673.

Canévet, G., Teghtsoonian, R., \& Teghtsoonian, M. (2003). A comparison of loudness change in signals that continuously rise and fall in amplitude. Acta Acustica United with Acustica, 89(3), 339-345.

Durlach, N. I., \& Braida, L. D. (1969). Intensity perception. I. Preliminary theory of intensity resolution. The Journal of the Acoustical Society of America, 46(2), 372-383.

Epstein, M. (2007). An introduction to induced loudness reduction. The Journal of the Acoustical Society of America, 122(3), EL74EL80.

Fletcher, H., \& Munson, W. A. (1933). Loudness, its definition, measurement and calculation. The Journal of the Acoustical Society of America, 5, 82-108.

Garner, W. R. (1958). Advantages of the discriminability criterion for a loudness scale. The Journal of the Acoustical Society of America, 30, 1005-1012.
Ghazanfar, A. A., Neuhoff, J. G., \& Logothetis, N. K. (2002). Auditory looming perception in rhesus monkeys. Proceedings of the National Academy of Sciences, 99(24), 15755-15757.

Gibson, J. J. (1966). The senses considered as perceptual systems. Boston: Houghton Mifflin.

Grassi, M., \& Darwin, C. J. (2006). The subjective duration of ramped and damped sounds. Perception \& Psychophysics, 68(8), 13821392.

Hall, D. A., \& Moore, D. R. (2003). Auditory neuroscience: The salience of looming sounds. Current Biology, 13, R91-R93.

Hartmann, W. M. (1998). Signals, sounds, and sensations. New York: Springer.

Helson, H. (1947). Adaptation-level as frame of reference for prediction of psychophysical data. The American Journal of Psychology, 60(1), 1-29.

Irino, T., \& Patterson, R. D. (1996). Temporal asymmetry in the auditory system. Journal of the Acoustic Society of America, 99 (4), 2316-2331.

Marks, L. E. (1988). Magnitude estimation and sensory matching. Perception \& Psychophysics, 43(6), 511-525.

Moore, B. C. J. (2004). Introduction to the psychology of hearing. New York: Academic Press.

Munson, W. A. (1947). The growth of auditory sensations. Journal of the Acoustic Society of America, 19, 584-591.

Neuhoff, J. G. (1998). Perceptual bias for rising tones. Nature, 395, $123-124$.

Neuhoff, J. G. (1999). Perception of changes in loudness. Nature, 398, 673-674.

Neuhoff, J. G. (2001). An adaptive bias in the perception of looming auditory motion. Ecological Psychology, 13(2), 87-110.

Pastore, R. E., Gaston, J. R., \& Berens, M. S. (2008). Backward recognition masking as a general type of interference in needed post-stimulus processing. Perception \& Psychophysics, 70(6), 1104-1116.

Plomp, R., \& Bouman, M. A. (1959). Relation between hearing threshold and duration for tone pulses. Journal of the Acoustic Society of America, 31, 749-758.

Poulton, E. C. (1968). The new psychophysics: Six models for magnitude estimation. Psychological Bulletin, 69(1), 1-19.

Poulton, E. C. (1969). Choice of first variables for single and repeated multiple estimates of loudness. Journal of Experimental Psychology, 80(2), 249-253.

Poulton, E. C. (1984). A linear relation between loudness and decibels. Perception \& Psychophysics, 36(4), 338-342.

Rosenblum, L. D., Carello, C., \& Pastore, R. A. (1987). Relative effectiveness of three stimulus variables for locating a moving sound source. Perception, 16, 175-186.

Rosenblum, L. D., Wuestefeld, A. P., \& Saldaña, H. M. (1993). Auditory looming perception: Influences on anticipatory judgments. Perception, 22, 1467-1482.

Scharf, B., Buus, S., \& Nieder, B. (2002). Loudness enhancement: Induced loudness reduction in disguise? Journal of the Acoustic Society of America, 112, 807-810.

Schlauch, R. S. (1992). A cognitive influence on the loudness of tones that change continuously in level. Journal of the Acoustic Society of America, 92(2), 758-765.

Seifritz, E., Neuhoff, J. G., Bilecen, D., Scheffler, K., Mustovic, H., Schächinger, H., \& Di Salle, F. (2002). Neural processing of auditory looming in the human brain. Current Biology, 12, $2147-2151$.

Stevens, S. S. (1935). The relation of pitch to intensity. The Journal of the Acoustical Society of America, 6, 150-154.

Stevens, S. S. (1955). The measurement of loudness. Journal of the Acoustic Society of America, 43, 405-416.

Stevens, S. S. (1956). The direct estimation of sensory magnitudesloudness. The American Journal of Psychology, 69, 1-25. 
Stevens, S. S. (1961). The psychophysics of sensory function. In W. A. Rosenblith (Ed.), Sensory communication. Cambridge MA: MIT Press.

Stevens, S. S., \& Davis, H. (1938). Hearing: It's psychology and physiology. Oxford: Wiley.

Stevens, S. S., \& Poulton, E. C. (1956). The estimation of loudness by unpracticed observers. Journal of Experimental Psychology, 51 (1), 71-78.

Teghtsoonian, M. (1965). The judgment of size. The American Journal of Psychology, 78(3), 392-402.

Teghtsoonian, R. (1973). Range effects in psychophysical scaling and a revision of Stevens' Law. The American Journal of Psychology, 86(1), 3-27.

Teghtsoonian, R., \& Teghtsoonian, M. (1986). Scaling loudness over short ranges: A reply to Poulton. Perception \& Psychophysics, 39 (1), 73-75.

Teghtsoonian, R., Teghtsoonian, M., \& Canévet, G. (2000). The perception of waning signals: Decruitment in loudness and perceived size. Perception \& Psychophysics, 62(3), 637-646.

Teghtsoonian, R., Teghtsoonian, M., \& Canévet, G. (2005). Sweepinduced acceleration in loudness change and the "bias for rising intensities". Perception \& Psychophysics, 67(4), 699712.

Wagner, E., \& Scharf, B. (2006). Induced loudness reduction as a function of exposure time and signal frequency. The Journal of the Acoustical Society of America, 119(2), 10121020.

Warren, R. M. (1958). A basis for judgments of sensory intensity. The American Journal of Psychology, 71, 675-687.

Warren, R. M. (1981). Measurement of sensory intensity. The Behavior and Brain Sciences, 4, 175-223.

Woodworth, R. S., \& Schlosberg, H. (1958). Experimental psychology. New York: Henry Holt.

Yost, W. A. (2007). Fundamentals of hearing. San Diego: Elsevier.

Zwicker, E., \& Fastl, H. (1990). Psychoacoustics - facts and models. Berlin: Springer.

Zwicker, E., Flottorp, G., \& Stevens, S. S. (1957). Critical band width in loudness summation. Journal of the Acoustic Society of America, 29, 548-557.

Zwislocki, J. J., \& Goodman, D. A. (1980). Absolute scaling of sensory magnitudes: A validation. Perception \& Psychophysics, 28(1), 28-38. 